\title{
Linguistic majorities with difference in support ${ }^{\frac{1}{4}}$
}

\author{
Patrizia Pérez-Asurmendi ${ }^{\mathrm{a}}$, Francisco Chiclana ${ }^{\mathrm{b}, \mathrm{c}, *}$ \\ ${ }^{a}$ PRESAD Research Group, SEED Research Group, IMUVA, Universidad de Valladolid, Valladolid, \\ Spain \\ ${ }^{b}$ Centre for Computational Intelligence, Faculty of Technology, De Montfort University, Leicester, UK \\ ${ }^{c}$ DMU Interdisciplinary Group in Intelligent Transport Systems, Faculty of Technology, De Montfort \\ University, Leicester, UK
}

\begin{abstract}
In social choice voting, majorities based on difference of votes and their extension, majorities based on difference in support, implement the crisp preference values (votes) and the intensities of preference provided by voters when comparing pairs of alternatives, respectively. The aim of these rules is declaring which alternative is socially preferred and to that, they require the winner alternative to reach a certain positive difference in its social valuation with respect to the one reached by the loser alternative. This paper introduces a new aggregation rule that extends majorities based on difference of votes from the context of crisp preferences to the framework of linguistic preferences. Under linguistic majorities with difference in support, voters express their intensities of preference between pairs of alternatives using linguistic labels and an alternative defeats another one when a specific support, fixed before the election process, is reached. There exist two main representation methodologies of linguistic preferences: the cardinal one based on the use of fuzzy sets, and the ordinal one based on the use of the 2-tuples. Linguistic majorities with difference in support are formalised in both representation settings, and conditions are given to guarantee that fuzzy linguistic majorities and 2-tuple linguistic majorities are mathematically isomorphic. Finally, linguistic majorities based on difference in support are proved to verify relevant normative properties: anonymity, neutrality, monotonicity, weak Pareto and cancellativeness.
\end{abstract}

Keywords: Social choice, Aggregation rule, Linguistic preferences, Linguistic majorities, Fuzzy sets, 2-tuples, Difference in support.

\section{Introduction}

Decision making problems deal with the social choice of the best alternative among all the possible alternatives taking into account the views and opinions, i.e. the preferences, of all the individuals of a particular social group [10,34, 38]. Two approaches are possible to address these problems $[24,26]$ : a direct approach that derives a social choice from the sole manipulation and processing of the information provided by all the individuals without the intermediate derivation of any kind of collective information using a fusion

\footnotetext{
Cite as: Patrizia Pérez-Asurmendi, Francisco Chiclana: Linguistic majorities with difference in support. Applied Soft Computing. 18, May 2014, Pages 196-208. doi: 10.1016/j.asoc.2014.01.010

*Corresponding author

Email addresses: patrizia.perez@eco.uva.es (Patrizia Pérez-Asurmendi), chiclana@dmu.ac.uk (Francisco Chiclana)
} 
or aggregation operator, which is characteristic of the indirect approach. Obviously, the type of aggregation rule implemented in the second approach is crucial in designing the corresponding social choice rule, and ultimately in the final social solution to the decision making problem. This paper deals with this specific issue, and it is devoted to the introduction of a new aggregation rule for individual preferences.

A comparison study between different alternative preference elicitation methods is reported in [32], where it was concluded that pairwise comparison methods are more accurate than non-pairwise methods. The main advantage of pairwise comparison methods is that facilitates individuals expressing their preferences because they focus exclusively on two alternatives at a time. Given two alternatives, an individual either prefers one to the other or is indifferent between them, which can be represented using a preference relation whose elements represent the preference of one alternative over another one. There exist two main mathematical models to represent pairwise comparison of alternatives based on the concept of preference relation $[10,35]$ : in the first one, a preference relation is defined for each one of the above three possible preference states, which is usually referred to as a preference structure on the set of alternatives; the second one integrates the three possible preference states into a single preference relation. This paper deals with the second type of relations, for which reciprocity of preferences is usually assumed in order to guarantee the following basic rationality properties in making paired comparisons [37]: indifference between any alternative and itself, and asymmetry of preferences, i.e. if an individual prefers alternative $x$ to $y$, that individual does not simultaneously prefer $y$ to $x$.

In classical voting systems the set of numerical values $\{1,0.5,0\}$, or its equivalent $\{1,0,-1\}[10]$, is used to represent when the first alternative is preferred to the second alternative, when both alternatives are considered equally preferred (indifference), and when the second alternative is preferred to the first one, respectively. This classical preference modelling constitutes the simplest numeric discrimination model of preferences, and it proves insufficient in many decision making situations as the following example illustrates: Let $x, y, z$ be three alternatives of which we know that one individual prefers $x$ to $y$ and $y$ to $z$, and another individual prefers $z$ to $y$ and $y$ to $x$; then using the above numerical values it may be difficult or impossible to decide which alternative is the best. As Fishburn points out in [10], if alternative $y$ is closer to the best alternative than to the worst one for both individuals then it might seem appropriate to 'elect' it as the social choice, whilst if it is closer to the worst than to the best, then it might be excluded from the choice set. Thus, in many cases it might be necessary the implementation of some kind of 'intensity of preference' between alternatives.

The concept of fuzzy set, which extends the classical concept of set, when applied to a classical relation leads to the concept of a fuzzy relation, which in turn allows the implementation of intensity of preferences [42]. In [2], we can find for the first time the fuzzy interpretation of intensity of preferences via the concept of a reciprocal fuzzy preference relation, which was later reinterpreted by Nurmi in [33]. In this approach, the numeric scale to evaluate intensity of preferences is the whole unit interval $[0,1]$ instead of $\{1,0.5,0\}$, which it is argued though to assume unlimited computational abilities and resources from the individuals [5].

Subjectivity, imprecision and vagueness in the articulation of opinions pervade real world decision applications, and individuals usually find difficult to evaluate their preference using exact numbers. Individuals might feel more comfortable using words by means of linguistic labels or terms to articulate their preferences [44]. Furthermore, hu- 
mans exhibit a remarkable capability to manipulate perceptions and other characteristics of physical and mental objects, without any exact numerical measurements and complex computations $[4,12,25,29,45]$. Therefore, in this paper, the individuals' preferences between pair of alternatives will be assumed to be given in the form of linguistic labels.

It was mentioned before that the type of aggregation rule implemented is crucial in designing the corresponding social choice rule. This paper focuses on the majority voting rules, which are very easy to understand by voters and therefore, when comparing two alternatives, they are seen as very attractive and appropriate to aggregate individual preferences into a collective one. Simple majority rule [31] stands out among the different majority rules. Under this rule, an alternative defeats another one when the number of votes cast for the first one exceeds the number of votes cast for the second one. In fact, the requirement to declare indifference between two alternatives is quite strong given that both alternatives have to receive exactly the same number of favourable votes. Furthermore, under the simple majority rule, the support required for an alternative to be the winner is minimum because it is only required to exceed the defeated alternative in just one vote. Being the most decisive aggregation rule turns out to become a drawback because the collective decision is very unstable, i.e. it could be reverted with the change of just one vote. In an attempt to overcome this shortcoming, tougher requirements for declaring an alternative as the winner have been defined and studied. Among these rules, it is worth mentioning the following: unanimous majority, absolute majority and qualified majorities [9, 10, 36].

Majorities based on difference of votes $\left(M_{k}\right)[14,23,27]$ constitute another general approach to majority voting rules. These majorities allow to calibrate the amount of support required for the winner alternative by means of a difference of votes fixed before the election process. At the extreme cases, i.e. no difference and maximum difference of votes, majorities based on difference of votes become the simple majority and unanimous majority, respectively. Moreover, if indifference is ruled out from individual preferences, they are equivalent to qualified majorities. With these rules, indifference between two alternatives is possible to be declared for more cases than under the simple majority rule. In fact, the indifference state could be enlarged as much as desired. The application of the majorities based on difference of votes to the case of $[0,1]$-valued reciprocal fuzzy preference relations is known as the majorities based on difference in support $\left(\widetilde{M}_{k}\right)[15]$.

The aim of this paper is to fill the gap between majorities based on difference of votes and majorities based on difference in support by providing new majority rules based on difference of support in the linguistic framework. Linguistic majorities with difference in support keep the essence of the former rules in the sense that for an alternative to be declared winner a specific support fixed before the election is to be achieved. The challenge here is to formally generalise the rules to the case of being the preferences linguistic rather than numeric in nature. An additional challenge here is to relate the linguistic majorities with difference in support that can be obtained when the main two approaches to model and represent linguistic information are applied. On the one hand, linguistic preferences can be modelled using a cardinal approach by means of fuzzy sets and their associated membership functions [42]. On the other hand, an ordinal approach can be used to model and manage linguistic preferences using the 2-tuple symbolic representation [21]. Therefore, two new and different linguistic majorities with difference in support will be introduced: the linguistic fuzzy majorities $\left(L M_{K}\right)$ and the 2-tuple linguistic majorities $\left(2 T M_{k}\right)$. Figure 1 illustrates the new linguistic majorities in relation with the corresponding ones developed for numerical preferences. 


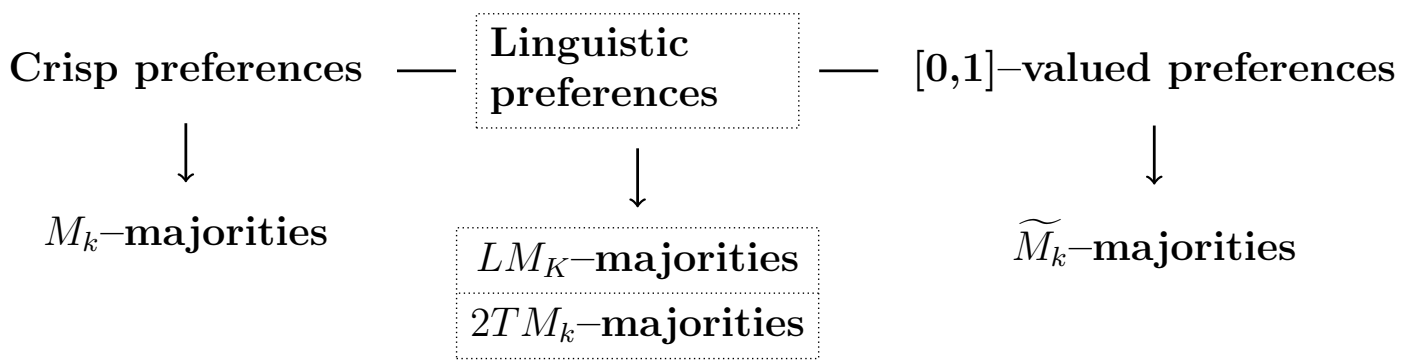

Figure 1: Preferences and majorities based on differences

The remainder of the paper is structured as follows: The next section introduces concepts essential to the understanding of the rest of the paper. Following that, Section 3 introduces the concept of linguistic majorities with difference in support and their mathematical formulation for the main two approaches to model and represent linguistic information: fuzzy set representation (Subsection 3.1) and the 2-tuple symbolic representation (Subsection 3.2). Section 4 proves that both linguistic majorities are mathematically isomorphic when fuzzy sets are defuzzified into their centroid. In Section 5, linguistic majorities based on difference in support are proved to verify the following relevant normative properties: anonymity, neutrality, monotonicity, weak Pareto and cancellativeness. Finally, in Section 6 conclusions are drawn and suggestions made for further work.

\section{Preliminaries}

Consider $m$ voters provide their preferences on pairs of alternatives of a set $X=$ $\left\{x_{1}, \ldots, x_{n}\right\}$. The preferences of each voter can be represented using a matrix, $R^{p}=$ $\left(r_{i j}^{p}\right)$, where $r_{i j}^{p}$ stands for the degree or intensity of preference of alternative $x_{i}$ over $x_{j}$ for voter $p$. The elements of $R^{p}$ can be numerical values or linguistic labels. In the following we focus on the former ones, leaving for Subsection 2.3 the second ones.

\subsection{Numeric preferences}

There are two main types of numeric preference relations: crisp preference relations and $[0,1]$-valued preference relations; with the second one being an extension of the first one, i.e. $[0,1]$-valued preference relations have crisp relations as a particular case.

1. A crisp preference relation is characterised for having elements $r_{i j}^{p}$ that belong to the discrete set of values $\{0,0.5,1\}$. In this context, when alternatives are pairwise compared, voters declare only their preference for one of the alternatives or their indifference between the two alternatives. Thus, if $r_{i j}^{p}=1$ then voter $p$ prefers alternative $x_{i}$ to alternative $x_{j}$, while if $r_{i j}^{p}=0.5$ the voter $p$ is indifferent between both alternatives. Moreover, it is always assumed that when $r_{i j}^{p}=0.5$ it is also $r_{j i}^{p}=0.5$; and when $r_{i j}^{p}=1$ then $r_{j i}^{p}=0$. This reciprocity property of preferences guarantees that preferences are represented by weak orderings, i.e. the asymmetric property is verified and 'inconsistent' situations where a voter could prefer two alternatives at the same time are avoided. Formally, a binary preference relation represented by $\succ_{p}$ is asymmetric if given two alternatives $x_{i}$ and $x_{j}, x_{i} \succ_{p} x_{j}$ implies that $x_{j} \nsucc_{p} x_{i}[11]$. 
2. The $[0,1]$-valued preference relation extends the crisp preference relation given that its elements $r_{i j}^{p}$ can take any value from the unit interval [0,1], with the following interpretation: $r_{i j}^{p}>0.5$ indicates that the individual $p$ prefers the alternative $x_{i}$ to the alternative $x_{j}$, with $r_{i j}^{p}=1$ being the maximum degree of preference for $x_{i}$ over $x_{j} ; r_{i j}^{p}=0.5$ represents indifference between $x_{i}$ and $x_{j}$ for voter $p$. As in the previous case, the reciprocity property of preferences, $r_{i j}^{p}+r_{j i}^{p}=1$, is usually assumed as an extension of the crisp asymmetry property described above. This type of preference relations will be referred to as reciprocal preference relations in this paper. In probabilistic choice theory, reciprocal preference relations are referred to as probabilistic binary preference relations. In fuzzy sets theory, reciprocal preference relations when used to represent intensities of preferences have usually been referred to as reciprocal fuzzy preference relations [30]. Reciprocal preference relations can be seen as a particular case of (weakly) complete fuzzy preference relations, i.e. fuzzy preference relations satisfying $r_{i j}+r_{j i} \geq 1 \forall i, j[12]$.

\subsection{Majorities based on differences}

In an attempt to overcome the support problems commonly attached to the simple majority rule in decision making contexts with crisp preferences, García-Lapresta and Llamazares [14] formalise the concept of majorities based on difference of votes or $M_{k}{ }^{-}$ majorities, which was later axiomatically characterised in [23, 27].

Definition 1 ( $M_{k}$-majorities). Given $k \in\{0, \ldots, m-1\}$, and a profile of individual crisp preferences $\left(R^{1}, \ldots, R^{m}\right)$ on a set of alternatives $X=\left\{x_{1}, \ldots, x_{n}\right\}$, the $M_{k}$-majority is a mapping from $X \times X$ to $\{1,0.5,0\}$, with the following expression:

$$
M_{k}\left(x_{i}, x_{j}\right)= \begin{cases}1 & \text { if } m_{i j}>m_{j i}+k \\ 0 & \text { if } m_{j i}>m_{i j}+k \\ 0.5 & \text { otherwise }\end{cases}
$$

where $m_{i j}$ is the number of votes cast by the individuals for the alternative $x_{i}$ when compared with alternative $x_{j}$ and $m_{j i}$ is the number of votes cast for the alternative $x_{j}$.

Thus, under $M_{k}$-majorities, given a difference of votes $k$, an alternative, $x_{i}$, defeats another alternative, $x_{j}$, by $k$ votes $\left(M_{k}\left(x_{i}, x_{j}\right)=1\right)$ when the difference between the votes cast for the alternative $x_{i}$ and the votes cast for the alternative $x_{j}$ is greater than $k$. Compared with the simple majority rule, the main change introduced by the majorities based on difference of votes affects the indifference state. The indifference of preference between two alternatives happens when the difference between the votes cast for both alternatives in absolute value is lower than or equal to $k$, i.e. when the difference of votes belongs to $\{0,1, \ldots, k\}$.

Example 1. Consider nine voters $(m=9)$ expressing their preferences between two alternatives, $\left(x_{1}, x_{2}\right)$, using the following profile of crisp preferences $(0,0,0,0,0,0.5,1,1,1)$. In this case, we observe that five voters prefer alternative $x_{2}\left(m_{21}=5\right)$, three voters prefer alternative $x_{1}\left(m_{12}=3\right)$, while one voter is indifferent between both alternatives. Under simple majority rule $x_{1}$ would not be the winner alternative $\left(m_{12}<m_{21}\right)$ but $x_{2}$. Simple majority rule corresponds by Definition 1 to $M_{0}$. We clearly see that under 
$M_{k}$-majorities $x_{1}$ is never declared the winner alternative (no matter the value of $k$ ), i.e. $M_{k}\left(x_{1}, x_{2}\right) \neq 1 \forall k$. Indeed, for this crisp preference profile we have the following:

$$
M_{k}\left(x_{1}, x_{2}\right)= \begin{cases}0 & \text { if } k \in\{0,1\} \\ 0.5 & \text { otherwise }\end{cases}
$$

Thus, when the difference of votes is set to be $k \in\{0,1\}$ we have that $x_{2}$ collectively defeats $x_{1}$, otherwise there exists a collective indifference between both alternatives. Unanimous majority rule requires that all voters prefer one alternative to the other one. In our example this is only possible when $\left(m_{12}, m_{21}\right) \in\{(9,0),(0,9)\}$, which corresponds to the $M_{k}$-majority rule with $k=8(=m-1)$.

$M_{k}$-majorities generalise other majority rules as the previous example has shown. Indeed, we have that $M_{0}$-majority is the simple majority rule, whereas $M_{(m-1)}$-majority is the unanimous majority rule. Moreover, $M_{k}$-majorities and qualified majorities, which are located between absolute majority and unanimity, are equivalent when individual indifference is ruled out from individual preferences [14]. These facts are summarised in Figure 2 .

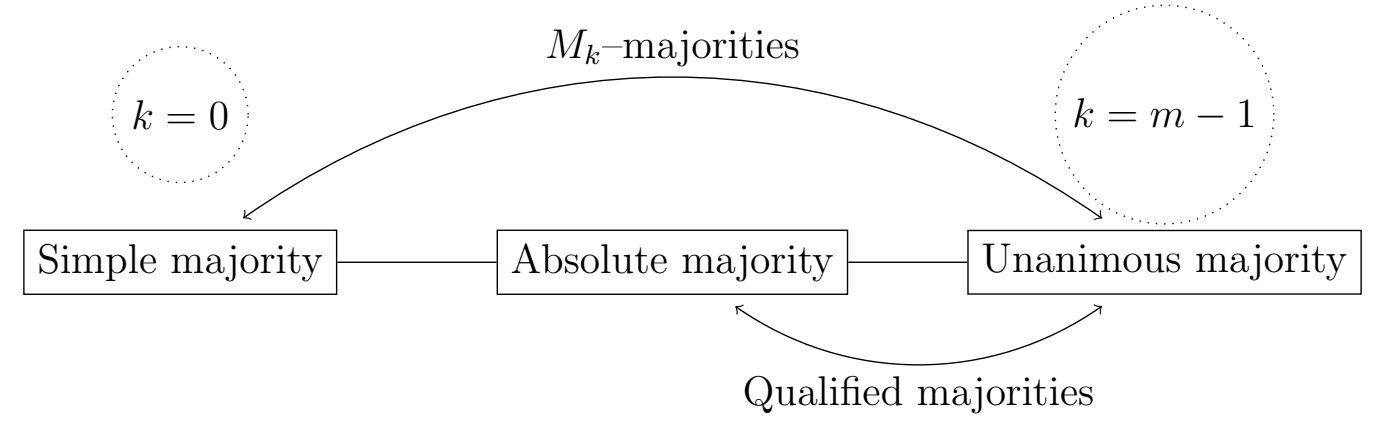

Figure 2: $M_{k}$-majorities versus other majorities

García-Lapresta and Llamazares [15] extend $M_{k}$-majorities to the framework of [0, 1]valued preferences. Majorities based on difference in support or $\widetilde{M}_{k}-$ majorities allow voters to show their preferences between pairs of alternatives through reciprocal preference relations whilst still maintaining the requirement of a higher support to the winner alternative than with the simple majority rule. Under $\widetilde{M}_{k}$-majorities, an alternative, $x_{i}$, defeats another one, $x_{j}$, by a threshold of support $k$, when the sum of the intensities of preference of $x_{i}$ over $x_{j}$ for the $m$ voters exceeds the sum of the intensities of preference of $x_{j}$ over $x_{i}$ in a quantity greater than $k$.

Definition 2 ( $\widetilde{M}_{k}-$ majorities). Given a threshold $k \in[0, m)$ and a profile of individual reciprocal preference relations $\left(R^{1}, \ldots, R^{m}\right)$, the $\widetilde{M}_{k}-$ majority is a mapping from $X \times X$ to $\{1,0.5,0\}$, with the following expression:

$$
\widetilde{M}_{k}\left(x_{i}, x_{j}\right)= \begin{cases}1 & \text { if } \sum_{p=1}^{m} r_{i j}^{p}>\sum_{p=1}^{m} r_{j i}^{p}+k \\ 0 & \text { if } \sum_{p=1}^{m} r_{j i}^{p}>\sum_{p=1}^{m} r_{i j}^{p}+k \\ 0.5 & \text { otherwise. }\end{cases}
$$


With $\widetilde{M}_{k}$-majorities, indifference between two alternatives happens when the difference in support between the alternatives in absolute value is lower than or equal to $k$, i.e. it is a value in the closed interval $[0, k]$.

A direct consequence of the reciprocity property is that $\widetilde{M}_{k}-$ majorities can be equivalently expressed in terms of the average of individual intensities of preference [15]:

$$
\widetilde{M}_{k}\left(x_{i}, x_{j}\right)= \begin{cases}1 & \text { if } \frac{1}{m} \sum_{p=1}^{m} r_{i j}^{p}>\frac{m+k}{2 m} \\ 0 & \text { if } \frac{1}{m} \sum_{p=1}^{m} r_{i j}^{p}<\frac{m-k}{2 m} \\ 0.5 & \text { otherwise. }\end{cases}
$$

The term $\frac{1}{m} \sum_{p=1}^{m} r_{i j}^{p}$ can be interpreted as the collective preference (the average of all the votes) of the first alternative, $x_{i}$, over the second one, $x_{j}$. Under the $\widetilde{M}_{k}-$ majorities, the indifference between two alternatives does not necessarily happen when the collective preference, expressed in terms of the arithmetic mean of the individual preferences, equals the value 0.5 . $\widetilde{M}_{k}-$ majorities declare indifference when the collective preference belongs to the closed interval $\left[0.5-\frac{k}{2 m}, 0.5+\frac{k}{2 m}\right]$, which we refer to as the indifference interval. When the collective preference is greater than the upper bound of the indifference interval, the first alternative is preferred to the second one. On the other hand, when the collective preference is lower than the lower bound of the indifference interval, the second alternative is preferred to the first one. In comparison with the simple majority rule, the $\widetilde{M}_{k}-$ majorities promote an increase on the cases where the collective indifference is declared, which depends on the threshold of support required to define the strict preference state.

Example 2. Consider the previous nine voters of Example 1 express their preferences between two alternatives, $\left(x_{1}, x_{2}\right)$, using the following profile of $[0,1]$-valued preferences $(0.1,0.25,0.4,0.4,0.4,0.5,0.9,0.9,0.9)$. This profile of $[0,1]$-valued preferences represents the same preference states as that of the profile of crisp preferences of Example 1: five voters prefer alternative $x_{2}$, three voters prefer alternative $x_{1}$, while one voter is indifferent between both alternatives. The solution to the equation

$$
\frac{1}{m} \sum_{p=1}^{m} r_{12}^{p}=\frac{m+k}{2 m} \Leftrightarrow \frac{4.75}{9}=\frac{9+k}{18}
$$

is $k=0.5$, and we have

$$
\widetilde{M}_{k}\left(x_{1}, x_{2}\right)= \begin{cases}1 & \text { if } k \in[0,0.5) \\ 0.5 & \text { otherwise }\end{cases}
$$

Notice that with the implementation of intensities of preference, $\widetilde{M}_{k}-$ majorities produce different collective preference outputs than the ones under $M_{k}$-majorities in the corresponding crisp preference case. Indeed, in this case alternative $x_{2}$ never defeats alternative $x_{1}$ and, on the contrary, alternative $x_{1}$ can defeat alternative $x_{2}$ if the difference in support is set to be lower than 0.5. This means that alternative $x_{1}$ would be the winner under simple majority rule, which is not the case in the corresponding crisp preference case of Example 1. It is worth mentioning here that three of the five experts that 
prefer alternative $x_{2}$ to alternative $x_{1}$ are indicating a slightly preference that is close to indifference. In contrast, the three voters preferring alternative $x_{1}$ over $x_{2}$ are doing this with an intensity degree close to the maximum. We observe that the implementation of intensities of preference in deriving majority preferences with difference in support allows for a more precise discrimination than if it were not taken into account.

\subsection{Linguistic preferences}

As mentioned before, subjectivity, imprecision and vagueness in the articulation of opinions pervade real world decision applications, and individuals might feel more comfortable using words by means of linguistic labels or terms to articulate their preferences [44]. In these cases is still valid the following quotation by Zadeh [45]: 'Since words, in general, are less precise than numbers, the concept of a linguistic variable serves the purpose of providing a means of approximate characterisation of phenomena which are too complex or too ill-defined to be amenable to description in conventional quantitative terms.'

Let $\mathcal{L}=\left\{l_{0}, \ldots, l_{s}\right\}$ be a set of linguistic labels $(s \geq 2)$, with semantic underlying a ranking relation that can be precisely captured with a linear order, i.e., $l_{0}<l_{1}<\cdots<$ $l_{s}$. Table 1 provides an example with seven linguistic labels and their corresponding semantic meanings for the comparison of the ordered pair of alternatives $\left(x_{i}, x_{j}\right)$.

\begin{tabular}{|c|l|}
\hline Linguistic label & Semantic meaning \\
\hline$l_{0}$ & $x_{j}$ is absolutely preferred to $x_{i}$ \\
$l_{1}$ & $x_{j}$ is highly preferred to $x_{i}$ \\
$l_{2}$ & $x_{j}$ is slightly preferred to $x_{i}$ \\
$l_{3}$ & $x_{i}$ and $x_{j}$ are equally preferred \\
$l_{4}$ & $x_{i}$ is slightly preferred to $x_{j}$ \\
$l_{5}$ & $x_{i}$ is highly preferred to $x_{j}$ \\
$l_{6}$ & $x_{i}$ is absolutely preferred to $x_{j}$ \\
\hline
\end{tabular}

Table 1: Seven linguistic labels and their semantic meanings

Assuming that the number of labels is odd and the central label $l_{s / 2}$ stands for the indifference state when comparing two alternatives, the remaining labels are usually located symmetrically around that central assessment, which guarantees that a kind of reciprocity property holds as in the case of numerical preferences previously discussed. Thus, if the linguistic assessment associated to the pair of alternatives $\left(x_{i}, x_{j}\right)$ is $l_{i j}=l_{h} \in \mathcal{L}$, then the linguistic assessment corresponding to the pair of alternatives $\left(x_{j}, x_{i}\right)$ would be $l_{j i}=l_{s-h}$. Therefore, the operator defined as $N\left(l_{h}\right)=l_{g}$ with $(g+h)=s$ is a negator operator because $N\left(N\left(l_{h}\right)\right)=N\left(l_{g}\right)=l_{h}$.

The corresponding matrix notation of linguistic individual preferences of voter $p$ is $R^{p}=\left(l_{i j}^{p}\right)$ with $l_{i j}^{p} \in \mathcal{L}$. A profile of linguistic preferences for the pair of alternatives $\left(x_{i}, x_{j}\right)$ is the vector of its associated linguistic preferences given by a set of $m$ voters, $\left(l_{i j}^{1}, \ldots, l_{i j}^{m}\right) \in \mathcal{L}^{m}$. The main two representation formats of linguistic information are [22]: the cardinal, which is based on the use of fuzzy set characterised with membership functions and that are mathematically processed using Zadeh's extension principle [44]; and the ordinal, which is based on the use of 2-tuples symbolic methodology [21]. 


\subsubsection{Fuzzy set linguistic representation format}

Convex normal fuzzy subsets of the real line, also known as fuzzy numbers, are commonly used to represent linguistic terms. By doing this, each linguistic assessment is represented using a fuzzy number that is characterised by a membership function, with base variable the unit interval $[0,1]$, describing its semantic meaning. The membership function maps each value in $[0,1]$ to a degree of performance representing its compatibility with the linguistic assessment [44]. Figure 3 illustrates a fuzzy number with Gaussian membership function.

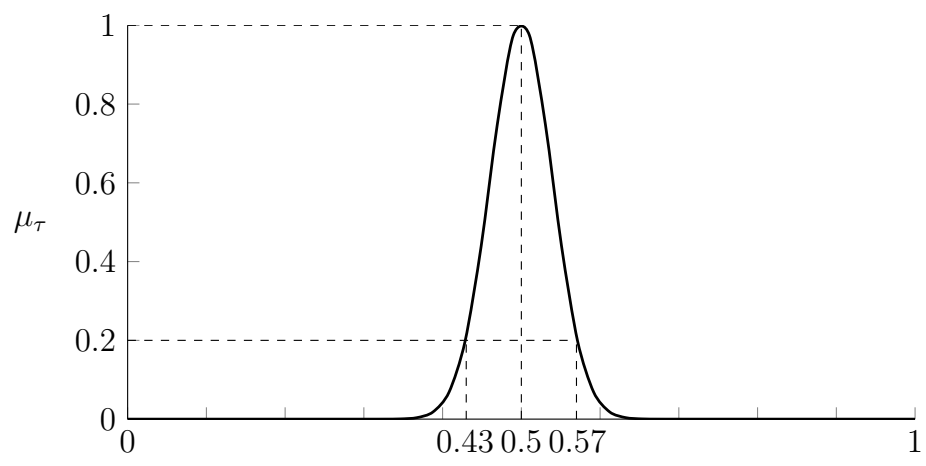

Figure 3: Representation of a fuzzy number with Gaussian membership function

It is worth mentioning that some authors consider trapezoidal fuzzy numbers as the most appropriate to represent linguistic preferences $[7,16]$ because they are more general than triangular and interval fuzzy numbers. Given four real numbers $t_{1}, t_{2}, t_{3}, t_{4}$, a trapezoidal fuzzy number $(\mathrm{TFN}) \tau=\left(t_{1}, t_{2}, t_{3}, t_{4}\right)$ is characterised by the following membership function:

$$
\mu_{\tau}(u)= \begin{cases}0 & \text { if } u \leq t_{1} \text { or } u \geq t_{4} \\ \frac{u-t_{1}}{t_{2}-t_{1}} & \text { if } t_{1}<u<t_{2} \\ \frac{t_{4}-u}{t_{4}-t_{3}} & \text { if } t_{2} \leq u \leq t_{3}\end{cases}
$$

A representation of a set of seven balanced linguistic terms using trapezoidal fuzzy numbers is given in Figure 4. Alternative representations are possible. For instance in [20], absolute preference of one alternative over another is represented using crisp values: $l_{0}=(0,0,0,0)$ and $l_{6}=(1,1,1,1)$.

\subsubsection{2-tuple linguistic representation format}

Linguistic assessments can also be represented and aggregated using symbolic representation models based on an ordinal interpretation of the semantic meaning associated to the linguistic labels. Within this framework, the following different approaches have being developed: a linguistic symbolic computational model based on ordinal scales and max-min operators [40], a linguistic symbolic computational model based on indexes $[8,39]$.

In Herrera and Martínez [21], a more general approach was introduced: the 2-tuple linguistic model. This linguistic model takes as a basis the symbolic representation model 


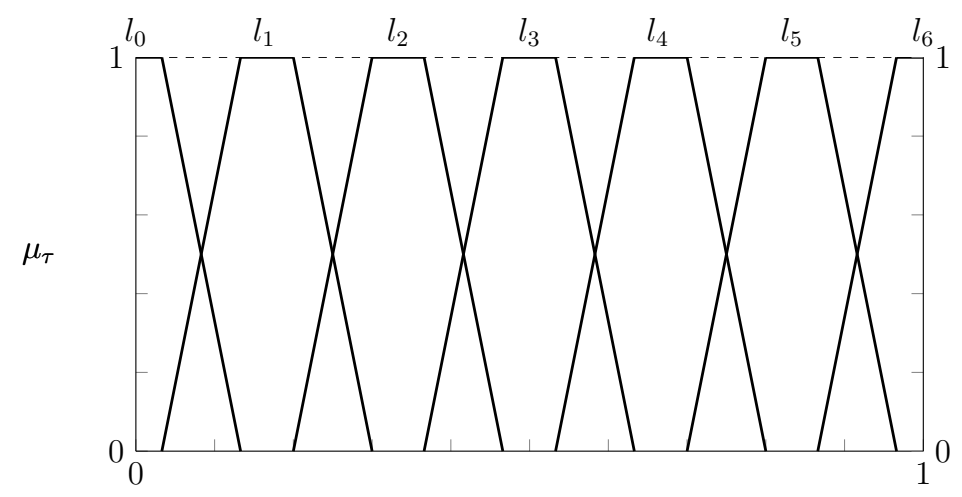

Figure 4: Representation of seven balanced linguistic terms with trapezoidal membership functions

based on indexes and in addition defines the concept of symbolic translation to represent the linguistic information by means of a pair of values called linguistic 2 -tuple, $\left(l_{b}, \lambda_{b}\right)$, where $l_{b} \in \mathcal{L}$ is one of the original linguistic terms and $\lambda_{b}$ is a numeric value representing the symbolic translation. This representation structure allows, on the one hand, to obtain the same information than with the symbolic representation model based on indexes without losing information in the aggregation phase. On the other hand, the result of the aggregation is expressed on the same domain as the one of the initial linguistic labels and therefore, the well-known re-translation problem of the above methods is avoided.

Definition 3 (Linguistic 2-tuple representation). Let $a \in[0, s]$ be the result of a symbolic aggregation of the indexes of a set of labels assessed in a linguistic term set $\mathcal{L}=\left\{l_{0}, \ldots, l_{s}\right\}$. Let $b=\operatorname{round}(a) \in\{0, \ldots, s\}$. The value $\lambda_{b}=a-b \in[-0.5,0.5)$ is called a symbolic translation, and the pair of values $\left(l_{b}, \lambda_{b}\right)$ is called the 2 -tuple linguistic representation of the symbolic aggregation $a$.

The 2-tuple linguistic representation of symbolic aggregation can be mathematically formalised with the following mapping:

$$
\begin{gathered}
\phi:[0, s] \longrightarrow \mathcal{L} \times[-0.5,0.5) \\
\phi(a)=\left(l_{b}, \lambda_{b}\right) .
\end{gathered}
$$

Based on the linear order of the linguistic term set and the complete ordering of the set $[-0.5,0.5)$, it is easy to prove that $\phi$ is strictly increasing and continuous and, therefore its inverse function exists:

$$
\begin{gathered}
\phi^{-1}: \mathcal{L} \times[-0.5,0.5) \longrightarrow[0, s] \\
\phi^{-1}\left(l_{b}, \lambda_{b}\right)=b+\lambda_{b}=a .
\end{gathered}
$$

The following negation operator is defined: $N(\phi(a))=\phi(s-a)$. Figure 5 illustrates the application of the 2-tuple function $\phi$ and its inverse for a linguistic term set of cardinality seven. The value of the symbolic translation is assumed to be 3.7, which means that $\operatorname{round}(3.7)=4$ and therefore it can be represented with the 2 -tuple $\left(l_{4},-0,3\right)$.

\section{Linguistic majorities with difference in support}

Before majorities based on difference in support in the context of linguistic preferences are defined, we need to introduce the linguistic decision rule concept. Recall that a profile of linguistic preferences for a pair of alternatives alternatives $\left(x_{i}, x_{j}\right)$ is a vector of its associated linguistic preferences given by a set of $m$ voters $\left(l_{i j}^{1}, \ldots, l_{i j}^{m}\right) \in \mathcal{L}^{m}$. 


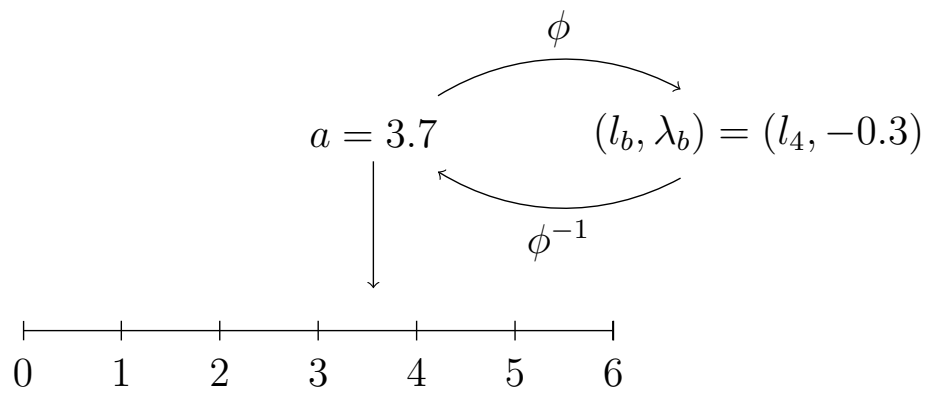

Figure 5: Ordinal linguistic representation: symbolic translation and 2-tuples

Definition 4. Given a pair of alternatives $\left(x_{i}, x_{j}\right) \in X \times X$, a linguistic decision rule is a mapping

$$
F: \mathcal{L}^{m} \longrightarrow\{0,0.5,1\}
$$

such that:

$$
F\left(l_{i j}^{1}, \ldots, l_{i j}^{m}\right)= \begin{cases}1 & \text { if } x_{i} \text { defeats } x_{j} \\ 0 & \text { if } x_{j} \text { defeats } x_{i} \\ 0.5 & \text { if } x_{i} \text { and } x_{j} \text { tie }\end{cases}
$$

The generalisation of the majorities based on difference of votes from the context of numerical preferences to the context of linguistic preferences involves: (1) the computation of the voters average linguistic assessment for a pair of alternatives, and (2) the evaluation of the difference between two linguistic evaluations. In the following, we will formalise this in both linguistic representation methodologies.

\subsection{Fuzzy linguistic majorities with difference in support}

In what follows, $\widetilde{A}_{i j}^{p}$ denotes the normal and convex fuzzy set representing the linguistic preference of alternative $x_{i}$ over $x_{j}$ provided by voter $p$. As mentioned before, the formalisation of the fuzzy linguistic majorities with difference in support requires the computation of the average fuzzy linguistic preference, $\frac{1}{m} \sum_{p=1}^{m} \widetilde{A}_{i j}^{p}$, of a profile of linguistic preferences $\left(\widetilde{A}_{i j}^{1}, \ldots, \widetilde{A}_{i j}^{m}\right)$.

The extension principle allows the domain of a functional mapping to be extended from crisp elements to fuzzy sets as given below [19, 44].

Definition 5 (Extension Principle). Let $X_{1} \times X_{2} \times \cdots \times X_{n}$ be a universal product set and $F$ a functional mapping of the form

$$
F: X_{1} \times X_{2} \times \cdots \times X_{n} \longrightarrow Y
$$

that maps the element $\left(x_{1}, x_{2}, \ldots, x_{n}\right) \in X_{1} \times X_{2} \times \cdots \times X_{n}$ to the element $y=$ $F\left(x_{1}, x_{2}, \ldots, x_{n}\right)$ of the universal set $Y$. Let $A_{i}$ be a fuzzy set over the universal set $X_{i}$ with membership function $\mu_{A_{i}}(i=1,2, \ldots, n)$. The membership function $\mu_{B}$ of the fuzzy set $B=F\left(A_{1}, \ldots, A_{n}\right)$ over the universal set $Y$ is

$$
\mu_{B}(y)= \begin{cases}\sup _{y=F\left(x_{1}, x_{2}, \ldots, x_{n}\right)}\left[\mu_{A_{1}}\left(x_{1}\right) * \mu_{A_{2}}\left(x_{2}\right) * \cdots * \mu_{A_{n}}\left(x_{n}\right)\right] \\ 0 & \text { if } \exists x_{1}, \ldots, x_{n}: y=F\left(x_{1}, \ldots, x_{n}\right) ; \\ 0 & \text { otherwise; }\end{cases}
$$


where $*$ is a t-norm.

For the work presented in this paper, the minimum t-norm $(\wedge)$ is used.

In what follows we will first extend the real function $f:[0,1] \times[0,1] \longrightarrow[0,1]$,

$$
f\left(u_{1}, u_{2}\right)=u_{1}+u_{2},
$$

to $f\left(\widetilde{A}_{1}, \widetilde{A}_{2}\right)$ where $\widetilde{A}_{1}, \widetilde{A}_{2}$ are fuzzy sets over the set $[0,1]$ with associated membership functions $\mu_{\widetilde{A}_{1}}, \mu_{\widetilde{A}_{2}}$. The extension principle states that $\widetilde{B}=f\left(\widetilde{A}_{1}, \widetilde{A}_{2}\right)$ is a fuzzy set over the set $[0,1]$ with membership function $\mu_{\widetilde{B}}:[0,1] \rightarrow[0,1]$;

$$
\mu_{\widetilde{B}}(u)=\sup _{\substack{u_{1}+u_{2}=u \\ u_{1}, u_{2} \in[0,1]}}\left[\mu_{\widetilde{A}_{1}}\left(u_{1}\right) \wedge \mu_{\widetilde{A}_{2}}\left(u_{2}\right)\right] .
$$

The representation theorem of fuzzy sets [44] provides an alternative and convenient way to define a fuzzy set via its corresponding family of crisp $\alpha$-level sets. The $\alpha$-level set of a fuzzy set $\widetilde{A}$ over the universe $Z$ is defined as $\widetilde{A}^{\alpha}=\left\{z \in Z \mid \mu_{\widetilde{A}}(z) \geq \alpha\right\}$. The set of crisp sets $\left\{\widetilde{A}^{\alpha} \mid 0<\alpha \leq 1\right\}$ is said to be a representation of the fuzzy set $\widetilde{A}$. Indeed, the fuzzy set $\widetilde{A}$ can be represented as

$$
\widetilde{A}=\underset{0<\alpha \leq 1}{\cup} \alpha \widetilde{A}^{\alpha}
$$

where $\alpha \widetilde{A}^{\alpha}$ is the scalar product of $\alpha$ with the set $\widetilde{A}^{\alpha}$. The membership function of $\widetilde{A}$ is as follows:

$$
\mu_{\widetilde{A}}(z)=\sup _{\alpha: z \in \widetilde{A}_{\alpha}} \alpha .
$$

More details about the representation theorem of fuzzy sets can be found in [44].

Let $\widetilde{A}_{1}^{\alpha}$ and $\widetilde{A}_{2}^{\alpha}$ be the $\alpha$-level sets of fuzzy sets $\widetilde{A}_{1}$ and $\widetilde{A}_{2}$ described above. We have

$$
f\left(\widetilde{A}_{1}^{\alpha}, \widetilde{A}_{2}^{\alpha}\right)=\left\{u_{1}+u_{2} \mid u_{1} \in \widetilde{A}_{1}^{\alpha}, u_{2} \in \widetilde{A}_{2}^{\alpha}\right\} .
$$

Both $\widetilde{B}^{\alpha}$ and $f\left(\widetilde{A}_{1}^{\alpha}, \widetilde{A}_{2}^{\alpha}\right)$ are crisp sets. Furthermore, as we prove next, we have the following equality:

$$
\widetilde{B}^{\alpha}=f\left(\widetilde{A}_{1}^{\alpha}, \widetilde{A}_{2}^{\alpha}\right)
$$

I. Let $u \in \widetilde{B}^{\alpha}$. By definition, we have $\mu_{\widetilde{B}}(u) \geq \alpha$ and there exist at least two values $u_{1}, u_{2} \in[0,1]$ such that $u_{1}+u_{2}=u$ and $\left[\mu_{\widetilde{A}_{1}}\left(u_{1}\right) \wedge \mu_{\widetilde{A}_{2}}\left(u_{2}\right)\right] \geq \alpha$. Therefore, it is true that $\mu_{\widetilde{A}_{1}}\left(u_{1}\right) \geq \alpha$ and $\mu_{\widetilde{A}_{2}}\left(u_{2}\right) \geq \alpha$, which means that $u_{1} \in \widetilde{A}_{1}^{\alpha}$ and $u_{2} \in \widetilde{A}_{2}^{\alpha}$. Consequently, $u \in f\left(\widetilde{A}_{1}^{\alpha}, \widetilde{A}_{2}^{\alpha}\right)$, i.e. $\widetilde{B}^{\alpha} \subseteq f\left(\widetilde{A}_{1}^{\alpha}, \widetilde{A}_{2}^{\alpha}\right)$.

II. Let $u \in f\left(\widetilde{A}_{1}^{\alpha}, \widetilde{A}_{2}^{\alpha}\right)$. There exist $u_{1} \in \widetilde{A}_{1}^{\alpha}$ and $u_{2} \in \widetilde{A}_{2}^{\alpha}$ such that $u_{1}+u_{2}=u$. We have that $\mu_{\widetilde{A}_{1}}\left(u_{1}\right) \geq \alpha$ and $\mu_{\widetilde{A}_{2}}\left(u_{2}\right) \geq \alpha$ and therefore it is:

$$
\sup _{\substack{u_{1}+u_{2}=u \\ u_{1} \in \widetilde{A}_{1}^{\alpha}, u_{2} \in \widetilde{A}_{2}^{\alpha}}}\left[\mu_{\widetilde{A}_{1}}\left(u_{1}\right) \wedge \mu_{\widetilde{A}_{2}}\left(u_{2}\right)\right] \geq \alpha .
$$


Because $\widetilde{A}_{1}^{\alpha}, \widetilde{A}_{2}^{\alpha} \subseteq[0,1]$, we have:

$$
\sup _{\substack{u_{1}+u_{2}=u \\ u_{1}, u_{2} \in[0,1]}}\left[\mu_{\widetilde{A}_{1}}\left(u_{1}\right) \wedge \mu_{\widetilde{A}_{2}}\left(u_{2}\right)\right] \geq \sup _{\substack{u_{1}+u_{2}=u \\ u_{1} \in \widetilde{A}_{1}^{\alpha}, u_{2} \in \widetilde{A}_{2}^{\alpha}}}\left[\mu_{\widetilde{A}_{1}}\left(u_{1}\right) \wedge \mu_{\widetilde{A}_{2}}\left(u_{2}\right)\right] .
$$

We conclude that $u \in \widetilde{B}^{\alpha}$, i.e. $f\left(\widetilde{A}_{1}^{\alpha}, \widetilde{A}_{2}^{\alpha}\right) \subseteq \widetilde{B}^{\alpha}$.

A similar reasoning will lead us to conclude that the $\alpha$-level set of the average of fuzzy numbers is equal to the average of the $\alpha$-level set of fuzzy sets [47]. Denoting $f\left(\widetilde{A}_{1}^{\alpha}, \widetilde{A}_{2}^{\alpha}\right)=\widetilde{A}_{1}^{\alpha}+\widetilde{A}_{2}^{\alpha}$, it is safe to use the following notation

$$
\widetilde{B}=\widetilde{A}_{1}+\widetilde{A}_{2} \Longleftrightarrow\left(\widetilde{A}_{1}+\widetilde{A}_{2}\right)^{\alpha}=\widetilde{A}_{1}^{\alpha}+\widetilde{A}_{2}^{\alpha}
$$

The $\alpha$-level sets of fuzzy numbers are closed intervals, and therefore interval arithmetic yields:

$$
\left(\widetilde{A}_{1}+\widetilde{A}_{2}\right)^{\alpha}=\widetilde{A}_{1}^{\alpha}+\widetilde{A}_{2}^{\alpha}=\left[u_{1}^{-}, u_{1}^{+}\right]+\left[u_{2}^{-}, u_{2}^{+}\right]=\left[u_{1}^{-}+u_{2}^{-}, u_{1}^{+}+u_{2}^{+}\right] .
$$

An example of the addition using the $\alpha$-level sets is shown in Figure 6. Given the fuzzy numbers $l_{3}$ and $l_{4}$ (Figure 4), $l_{3}+l_{4}$ is constructed by applying (6) to compute the lower and upper bounds of its $\alpha$-level sets, followed by the application of the representation theorem of fuzzy sets. The computation of the lower bound of the $0.2-$ level set is given.

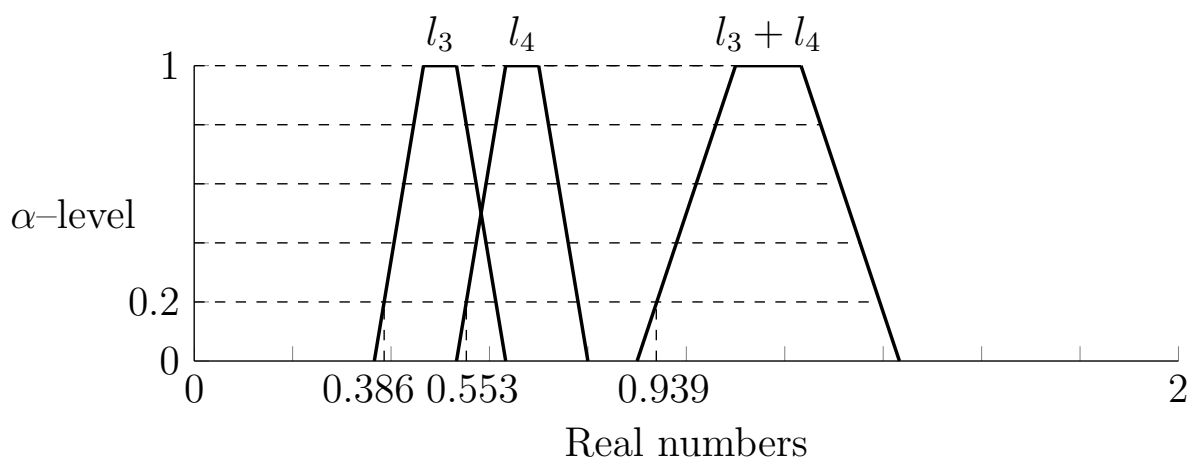

Figure 6: $\alpha$-level addition of linguistic terms

Once we have solved the computation of the average linguistic preference of the profile of linguistic preferences associated to a pair of alternatives, the formalisation of fuzzy linguistic majorities with difference in support requires its classification regarding its containment in one of the intervals corresponding to the social preference or social indifference established by the value of $k$. In other words, we need to find out when the following inequality $\frac{1}{m} \sum_{p=1}^{m} \widetilde{A}_{i j}^{p}>\frac{m+k}{2 m}$ is true or when it is false. Because crisp numbers are particular types of fuzzy numbers, the above inequality involves the comparison of fuzzy numbers. Yager [41] pointed out that this problem has been extensively studied and that there is no unique best approach. Indeed, the set of fuzzy numbers is not totally ordered and therefore it is not possible to achieve a clear social decision in this case. This 
is clearly illustrated in Figure 7, where two different aggregated fuzzy set are displayed, namely $\widetilde{B}_{1}$ and $\widetilde{B}_{2}$. Because $\widetilde{B}_{1}$ completely belongs to one of the intervals of preference there is no doubt about the social decision, which in the illustrated case implies that alternative $x_{j}$ defeats alternative $x_{i}$ with a different in support $k$. A similar conclusion would derive if the fuzzy set complete belongs the interval of indifference between both alternatives. On the contrary, the case represented by $\widetilde{B}_{2}$ is ambiguous given that such set is located in between the interval of preference for $x_{i}$ and the indifference state. Thus, a different approach is needed if we are to provide a clear cut social choice as per Definition 4 .
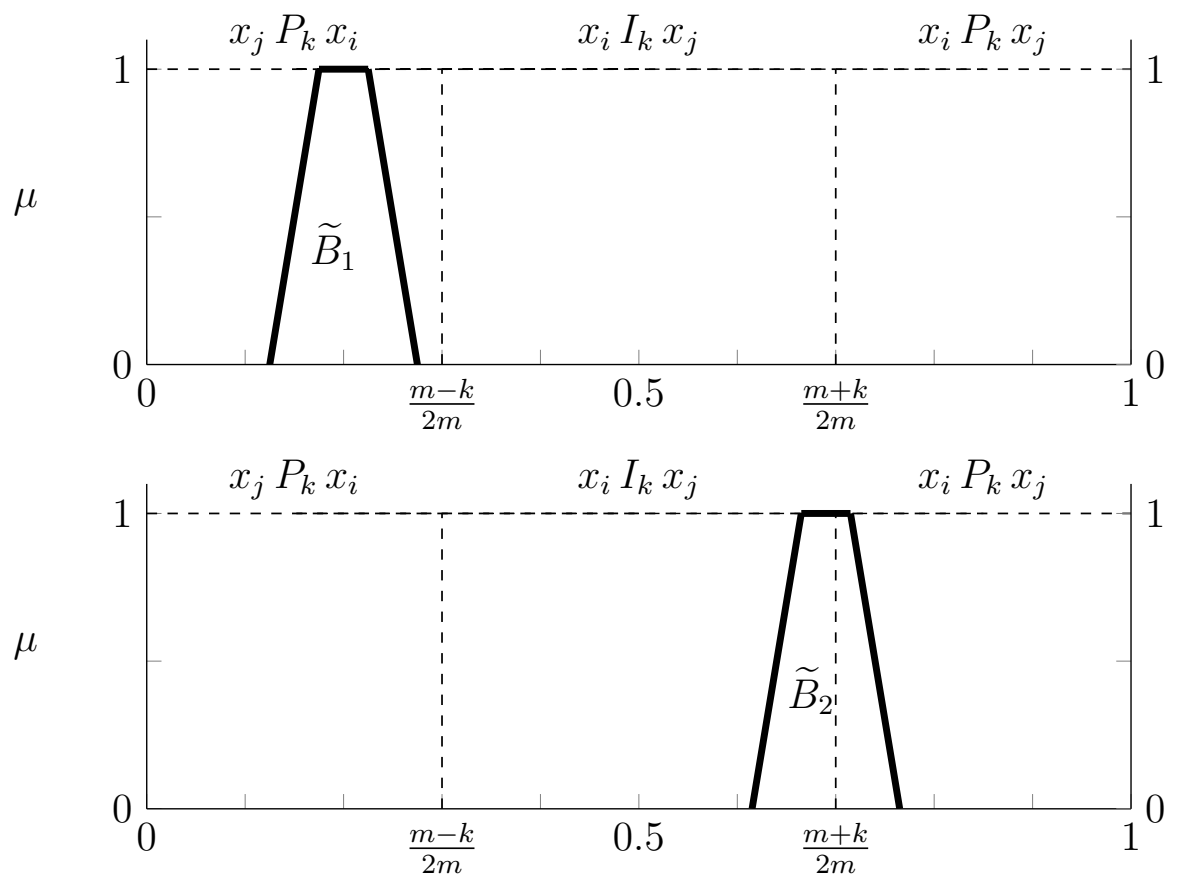

Figure 7: Comparison between aggregated fuzzy sets and preference-indifference states

A widely used approach to rank fuzzy numbers consists in converting them into a representative crisp value, and perform the comparison on them, a methodology originally proposed by Zadeh in [43]. This approach has been proposed and used in the selection process of decision making problems under uncertainty where ranking of fuzzy or intuitionistic fuzzy sets is a must to arrive at a decision [41]. Recently, a study by Brunelli and Mezei [3] that compares different ranking methods for fuzzy numbers concludes that 'it is impossible to give a final answer to the question on what ranking method is the best. Most of the time choosing a method rather than another is a matter of preference or is context dependent.' Two defuzzification methods widely used in fuzzy set theory are: the centre of area method (COA) and the mean of maximum method (MOM). The first one computes the centre of mass of the membership function of the fuzzy set (the centroid), whereas the second one computes the mid-point of the 1-level set of the fuzzy set. The COA method maintains the underlying semantic ranking relation within the set of linguistic labels as discussed before, i.e. given two linguistic labels $l_{i}, l_{j} \in \mathcal{L}$ such that $l_{i}<l_{j}$ then $\boldsymbol{u}_{C O A}\left(l_{i}\right)<\boldsymbol{u}_{C O A}\left(l_{j}\right)$, and therefore its use is proposed in the approach presented here. It is worth mentioning Brunelli and Mezei's correlation study, and their centrality analysis associated to the corresponding correlation network representation, 
which shows the centre of area method as one of the highest central defuzzification methods. Furthermore, for a trapezoidal fuzzy number $\widetilde{A}$ with membership function (2), we have $\boldsymbol{u}_{C O A}(\widetilde{A})=\frac{t_{1}+t_{4}}{2}$ and under the assumed property of internal symmetry of the linguistic labels it is $\boldsymbol{u}_{C O A} \equiv \boldsymbol{u}_{M O M}$. From now on, we refer to the centre of area value of fuzzy number $\widetilde{A}$ simply as $\boldsymbol{u}(\widetilde{A})$. Given two trapezoidal fuzzy numbers, namely $\widetilde{A}_{1}$ and $\widetilde{A}_{2}$, it holds that $\boldsymbol{u}\left(\widetilde{A}_{1}+\widetilde{A}_{2}\right)=\boldsymbol{u}\left(\widetilde{A}_{1}\right)+\boldsymbol{u}\left(\widetilde{A}_{2}\right)$. Hence, $\boldsymbol{u}$ is an additive function.

The range of function $\boldsymbol{u}$ is $\left[\boldsymbol{u}\left(l_{0}\right), \boldsymbol{u}\left(l_{s}\right)\right]$, while the range of $\frac{m+k}{2 m}$ is $[0,1]$. Thus, to carry out a fair comparison in the formalisation of the linguistic majority with difference in support, the following function $\boldsymbol{v}$ with range $[0,1]$ is used:

$$
\boldsymbol{v}(\widetilde{A})=\frac{\boldsymbol{u}(\widetilde{A})-\boldsymbol{u}\left(l_{0}\right)}{\boldsymbol{u}\left(l_{s}\right)-\boldsymbol{u}\left(l_{0}\right)} .
$$

Below, we formally define linguistic majorities with difference in support represented by fuzzy sets. Under these rules, an alternative, $x_{i}$, defeats another one, $x_{j}$, by a threshold of support $K$, if the defuzzified value of the average fuzzy set of the voters' linguistic valuations between $x_{i}$ and $x_{j}$ exceeds the value 0.5 in a quantity that depends on the threshold $K$, fixed before the election process.

Definition 6 ( $L M_{K}$-majorities with difference in support). Given a set of alternatives $X$ and a profile of individual reciprocal fuzzy linguistic preference relations $R(X)=\left(R^{1}, \ldots, R^{m}\right)$, the $L M_{K}$-majorities with difference in support are the following linguistic decision rules:

$$
L M_{K}\left(\widetilde{A}_{i j}^{1}, \ldots, \widetilde{A}_{i j}^{m}\right)= \begin{cases}1 & \text { if } \boldsymbol{v}\left(\frac{1}{m} \sum_{p=1}^{m} \widetilde{A}_{i j}^{p}\right)>\frac{m+K}{2 m} \\ 0 & \text { if } \boldsymbol{v}\left(\frac{1}{m} \sum_{p=1}^{m} \widetilde{A}_{i j}^{p}\right)<\frac{m-K}{2 m} \\ 0.5 & \text { otherwise }\end{cases}
$$

where $\boldsymbol{v}\left(\frac{1}{m} \sum_{p=1}^{m} \widetilde{A}_{i j}^{p}\right)$ is the defuzzified value of the fuzzy average linguistic preference of the profile of fuzzy linguistic preferences of the pair of alternatives $\left(x_{i}, x_{j}\right)$; and $K \in[0, m)$ represents the threshold of support required for an alternative to be the social winner.

In the following result we prove that function $\boldsymbol{v}$ is additive.

Proposition 1. Function $\boldsymbol{v}$ verifies

$$
\boldsymbol{v}\left(\frac{1}{m} \sum_{p=1}^{m} \widetilde{A}_{i j}^{p}\right)=\frac{1}{m} \sum_{p=1}^{m} \boldsymbol{v}\left(\widetilde{A}_{i j}^{p}\right) .
$$

Proof. Because $\boldsymbol{u}$ is additive we have that

$$
\boldsymbol{u}\left(\frac{1}{m} \sum_{p=1}^{m} \widetilde{A}_{i j}^{p}\right)=\frac{1}{m} \sum_{p=1}^{m} \boldsymbol{u}\left(\widetilde{A}_{i j}^{p}\right) .
$$


Also, we have that $\boldsymbol{u}$ and $\boldsymbol{v}$ are related in the form $\boldsymbol{u}=c \cdot \boldsymbol{v}+d$ where $c=\boldsymbol{u}\left(l_{s}\right)-\boldsymbol{u}\left(l_{0}\right)$ and $d=\boldsymbol{u}\left(l_{0}\right)$, it is:

$$
\begin{aligned}
& \boldsymbol{u}\left(\frac{1}{m} \sum_{p=1}^{m} \widetilde{A}_{i j}^{p}\right)=c \cdot \boldsymbol{v}\left(\frac{1}{m} \sum_{p=1}^{m} \widetilde{A}_{i j}^{p}\right)+d \\
& \boldsymbol{u}\left(\frac{1}{m} \sum_{p=1}^{m} \widetilde{A}_{i j}^{p}\right)=\frac{1}{m} \sum_{p=1}^{m} \boldsymbol{u}\left(\widetilde{A}_{i j}^{p}\right)=\frac{1}{m} \sum_{p=1}^{m}\left[c \cdot \boldsymbol{v}\left(\widetilde{A}_{i j}^{p}\right)+d\right]=c \cdot \frac{1}{m} \sum_{p=1}^{m} \boldsymbol{v}\left(\widetilde{A}_{i j}^{p}\right)+d .
\end{aligned}
$$

Thus, we have:

$$
\boldsymbol{v}\left(\frac{1}{m} \sum_{p=1}^{m} \widetilde{A}_{i j}^{p}\right)=\frac{1}{m} \sum_{p=1}^{m} \boldsymbol{v}\left(\widetilde{A}_{i j}^{p}\right)
$$

i.e. $\boldsymbol{v}$ is additive.

Expression (7) can be rewritten as follows:

$$
L M_{K}\left(\widetilde{A}_{i j}^{1}, \ldots, \widetilde{A}_{i j}^{m}\right)= \begin{cases}1 & \text { if } \frac{1}{m} \sum_{p=1}^{m} \boldsymbol{v}\left(\widetilde{A}_{i j}^{p}\right)>\frac{m+K}{2 m} \\ 0 & \text { if } \frac{1}{m} \sum_{p=1}^{m} \boldsymbol{v}\left(\widetilde{A}_{i j}^{p}\right)<\frac{m-K}{2 m} \\ 0.5 & \text { otherwise }\end{cases}
$$

where $K \in[0, m)$ and $\frac{1}{m} \sum_{p=1}^{m} \boldsymbol{v}\left(\widetilde{A}_{i j}^{p}\right)$ is the average of the defuzzified values associated with the profile of fuzzy linguistic preferences of the pair of alternatives $\left(x_{i}, x_{j}\right)$ as per the assessment of each individual voter.

It was mentioned before that the linguistic labels are located symmetrically around the central label $l_{s / 2}$ and therefore it is also appropriate to assume that the membership functions defining the fuzzy linguistic labels result in centroids symmetrically distributed with respect to the centroid of the central label. Because the central fuzzy linguistic label $l_{s / 2}$ stands for the indifference state when comparing two alternatives, it is also appropriate to have 0.5 as its centroid. Thus, denoting the centroid of fuzzy linguistic label $l_{k}$ by $\boldsymbol{u}\left(l_{h}\right)$ we have that $\boldsymbol{u}\left(l_{0}\right)+\boldsymbol{u}\left(l_{s}\right)=1$ and

$$
\boldsymbol{u}\left(l_{h}\right)=\boldsymbol{u}\left(l_{0}\right)+h \cdot \frac{1-2 \cdot \boldsymbol{u}\left(l_{0}\right)}{s},
$$

which guarantees an evenly distribution of the centroids of the fuzzy linguistic labels around the value 0.5 . In this context, the application of function $\boldsymbol{v}$ results in

$$
\boldsymbol{v}\left(l_{h}\right)=\frac{h}{s} .
$$

In the following, we provide an example to illustrate the application of the $L M_{K^{-}}$ majorities with difference in support.

Example 3. Consider nine voters expressing their preferences between two alternatives, $\left(x_{1}, x_{2}\right)$, using the linguistic labels of Table 1 represented by a set of balanced trapezoidal fuzzy numbers as illustrated in Figure 4 and verifying (9). Assume the voters provide the fuzzy linguistic profile of Table 2 . 


\begin{tabular}{|c|c|}
\hline Linguistic label & $\boldsymbol{v}\left(l_{h}\right)$ \\
\hline$l_{0}$ & 0 \\
$l_{1}$ & $1 / 6$ \\
$l_{2}$ & $1 / 3$ \\
$l_{3}$ & 0.5 \\
$l_{4}$ & $2 / 3$ \\
$l_{5}$ & $5 / 6$ \\
$l_{6}$ & 1 \\
\hline
\end{tabular}

Table 2: Linguistic labels and centroids of their fuzzy trapezoidal membership functions

In Table 3 two different $L M_{K}$-majorities with difference in support are computed: the simple linguistic majority $L M_{0}$, and $L M_{3}$.

\begin{tabular}{|c|c|c|c|}
\hline$K$ & $\boldsymbol{v}\left(\frac{1}{m} \sum_{p=1}^{m} \widetilde{A}_{12}^{p}\right)=\frac{1}{m} \sum_{p=1}^{m} \boldsymbol{v}\left(\widetilde{A}_{12}^{p}\right)$ & $\frac{m+K}{2 m}$ & $L M_{K}$ \\
\hline 0 & $\boldsymbol{v}\left(\frac{l_{0}+l_{1}+l_{2}+l_{2}+l_{2}+l_{3}+l_{6}+l_{6}+l_{6}}{9}\right)=14 / 27$ & 0.5 & 1 \\
3 & $9 / 3$ & 0.5 \\
\hline
\end{tabular}

Table 3: Aggregation and results for two different $L M_{K}-$ majorities

In the first case, it is enough to have an average centroid of the fuzzy linguistic profile greater than the centroid (0.5) of the central fuzzy linguistic assessment $\left(l_{3}\right)$ to declare $x_{1}$ the social winner alternative. In the second case, the threshold required implies that the average centroid of the fuzzy linguistic profile is to be greater than the centroid of the fuzzy linguistic label $l_{4}$ for $x_{1}$ to be declared the social winner alternative. In the first case, we have that $x_{1}$ can indeed be declared the social $L M_{0}$ winner alternative, whilst there is social $L M_{3}$ indifference in the second case.

The $L M_{K}$-majorities with difference in support for this particular fuzzy linguistic profile have an expression similar to the one achieved for the case of $[0,1]$-valued preferences, being as follows:

$$
\operatorname{LM}_{K}\left(l_{0}, l_{1}, l_{2}, l_{2}, l_{2}, l_{3}, l_{6}, l_{6}, l_{6}\right)= \begin{cases}1 & \text { if } K \in[0,1 / 3) \\ 0.5 & \text { otherwise }\end{cases}
$$

The following observations are worth highlighting:

(i) $L M_{K}$-majorities with difference in support generalise the simple linguistic majority [13]. Indeed, $L M_{0}$-majority coincides with the simple majority based on linguistic labels. In this case, no difference of support between the alternatives is required.

(ii) Linguistic unanimity holds when all the voters involved in the election prefer the same alternative, even when their intensities of preference could differ from one to another. The following three linguistic profiles with nine voters and a set of seven linguistic terms will serve to illustrate this concept.

$$
\left(l_{0}, l_{0}, l_{0}, l_{1}, l_{1}, l_{1}, l_{2}, l_{2}, l_{2}\right) ;\left(l_{6}, l_{6}, l_{6}, l_{6}, l_{6}, l_{6}, l_{6}, l_{6}, l_{6}\right) ;\left(l_{0}, l_{0}, l_{0}, l_{0}, l_{0}, l_{0}, l_{0}, l_{0}, l_{3}\right) .
$$


The first two profiles fulfill linguistic unanimity: in the first one all nine voters express a preference for the second alternative, whilst in the second one the first alternative is preferred by all nine voters. However, in the third profile there is no unanimity of preferences because voter 9 expresses indifference between both alternatives and therefore differs from the rest of voters, who strongly prefer the second alternative.

Given a profile of fuzzy linguistic preferences $\left(\widetilde{A}_{i j}^{1}, \ldots, \widetilde{A}_{i j}^{m}\right)$, linguistic unanimity happens if $\boldsymbol{v}\left(\widetilde{A}_{i j}^{p}\right) \leq \boldsymbol{v}\left(l_{\frac{s}{2}-1}\right) \forall p$, or $\boldsymbol{v}\left(\widetilde{A}_{i j}^{p}\right) \geq \boldsymbol{v}\left(l_{\frac{s}{2}+1}\right) \forall p$. In the first case, all voters prefer the second alternative over the first one, whilst the first alternative is preferred over the second one in the second case. Algebraic manipulation leads us to the following threshold values: $K<m-2 m \cdot \boldsymbol{v}\left(l_{\frac{s}{2}-1}\right)$ for the social preference of the second alternative, and $K<2 m \cdot \boldsymbol{v}\left(l_{\frac{s}{2}+1}\right)-m$ for the social preference of the first alternative.

Because we are assuming that the linguistic labels are symmetrical and balanced around the central one, then if the fuzzy sets used to represent them are all of the same type and uniformly distributed in the domain $[0,1]$, the normalised centroid function $\boldsymbol{v}$ would be $\boldsymbol{v}\left(l_{h}\right)=h / s \forall h$, and therefore the threshold value to assure linguistic unanimity would be $K<2 \mathrm{~m} / \mathrm{s}$.

\subsection{2-tuple linguistic majorities with difference in support}

In order to extend the $M_{k}$-majorities to the framework of the 2-tuple, the addition as well as a rule to compare 2 -tuples are needed.

Definition 7 (2-tuple Addition [21]). The addition of 2-tuples, $\phi\left(a_{1}\right)=\left(l_{b_{1}}, \lambda_{b_{1}}\right)$ and $\phi\left(a_{2}\right)=\left(l_{b_{2}}, \lambda_{b_{2}}\right)$, with $b_{1}=\operatorname{round}\left(a_{1}\right), b_{2}=\operatorname{round}\left(a_{2}\right), \lambda_{b_{1}}=a_{1}-b_{1}$ and $\lambda_{b_{2}}=a_{2}-b_{2}$, is computed as follows:

$$
\phi\left(a_{1}\right)+\phi\left(a_{2}\right)=\left(l_{b_{12}}, \lambda_{b_{12}}\right)
$$

with $b_{12}=\operatorname{round}\left(a_{1}+a_{2}\right)$, and $\lambda_{b_{12}}=\left(a_{1}+a_{2}\right)-b_{12}$.

Definition 8 (2-tuple Lexicographic Ordering [21]). Given $\phi\left(a_{1}\right)=\left(l_{b_{1}}, \lambda_{b_{1}}\right)$ and $\phi\left(a_{2}\right)=\left(l_{b_{2}}, \lambda_{b_{2}}\right)$, we have that:

1. if $b_{1}$ is greater than $b_{2}$, then $\phi\left(a_{1}\right)>\phi\left(a_{2}\right)$.

2. if $b_{1}$ is equal to $b_{2}$ and $\lambda_{b_{1}}$ is greater than $\lambda_{b_{1}}$, then $\phi\left(a_{1}\right)>\phi\left(a_{2}\right)$.

3. if $b_{1}$ is equal to $b_{2}$ and $\lambda_{b_{1}}$ is equal to $\lambda_{b_{1}}$, then $\phi\left(a_{1}\right)=\phi\left(a_{2}\right)$.

Below, we formally define 2-tuple linguistic majorities with difference in support. Under these rules, an alternative, $x_{i}$, defeats another one, $x_{j}$, by a threshold of support $k$, if the 2-tuple linguistic representation of the average symbolic aggregation of the linguistic preferences of $x_{i}$ over $x_{j}$ exceeds the 2-tuple linguistic representation associated to the indifference state in a value that depends on the threshold $k$, fixed before the election process. 
Definition 9 ( $2 T M_{k}$-majorities with difference in support). Given a set of alternatives $X$ and a profile of individual reciprocal 2-tuple linguistic preference relations $\left(R^{1}, \ldots, R^{m}\right)$, the $2 T M_{k}-$ majority with difference in support is the following linguistic decision rule:

$$
2 T M_{k}\left(a_{i j}^{1}, \ldots, a_{i j}^{m}\right)= \begin{cases}1 & \text { if } \frac{1}{m} \sum_{p=1}^{m} \phi\left(a_{i j}^{p}\right)>\phi\left(\frac{s \cdot m+k}{2 m}\right) \\ 0 & \text { if } \frac{1}{m} \sum_{p=1}^{m} \phi\left(a_{i j}^{p}\right)<\phi\left(\frac{s \cdot m-k}{2 m}\right) \\ 0.5 & \text { otherwise }\end{cases}
$$

where $\frac{1}{m} \sum_{p=1}^{m} \phi\left(a_{i j}^{p}\right)$ is the average of the 2-tuple representation of the linguistic preferences provided by the voters for the pair of alternatives $\left(x_{i}, x_{j}\right), \phi$ is the 2 -tuple symbolic aggregation mapping (3); and $k \in[0, m \cdot s)$ represents the threshold of support required for an alternative to be the social winner.

In the context of the 2 -tuple linguistic representation, the linguistic label $l_{h}$ is associated a valuation that coincides with its ordering position within $\mathcal{L}$, i.e. $h$, and therefore the maximum social preference value a set of voters can assign to an alternative when compared against another one is $m \cdot s$, which corresponds to the linguistic profile $\left(l_{s}, \ldots, l_{s}\right)$. This explains why $[0, m \cdot s)$ is the range of values for parameter $k$.

Given that in the ordinal representation of linguistic information the addition of linguistic labels is defined as $l_{a_{1}}+l_{a_{2}}=l_{a_{1}+a_{2}}$ [39], it is obvious that function $\phi$ is additive. Therefore expression (10) can be rewritten as follows:

$$
2 T M_{k}\left(a_{i j}^{1}, \ldots, a_{i j}^{m}\right)= \begin{cases}1 & \text { if } \phi\left(\frac{1}{m} \sum_{p=1}^{m} a_{i j}^{p}\right)>\phi\left(\frac{s \cdot m+k}{2 m}\right) ; \\ 0 & \text { if } \phi\left(\frac{1}{m} \sum_{p=1}^{m} a_{i j}^{p}\right)<\phi\left(\frac{s \cdot m-k}{2 m}\right) ; \\ 0.5 & \text { otherwise }\end{cases}
$$

where $\frac{1}{m} \sum_{p=1}^{m} a_{i j}^{p}$ is the symbolic aggregation, specifically the arithmetic mean, of the linguistic preferences provided by the voters for the pair of alternatives $\left(x_{i}, x_{j}\right)$.

The following example illustrates the use of the $2 T M_{k}$-majorities with difference in support.

Example 4 (Example 3 continuation). Table 4 presents the results for two different $2 T M_{k}$-majorities: $2 T M_{0}$ and $2 T M_{18}$. In the first one, the alternative $x_{1}$ is declared the winner when the 2-tuple representation of the symbolic arithmetic mean of the linguistic preferences provided by the voters for the pair of alternatives $\left(x_{1}, x_{2}\right)$ is greater than the indifference 2 -tuple $\left(l_{3}, 0\right)$; while it has to be greater than the 2 -tuple $\left(l_{4}, 0\right)$ in the second case.

The $2 T M_{k}$-majorities with difference in support for this particular linguistic profile is as follows:

$$
2 T M_{k}\left(l_{0}, l_{1}, l_{2}, l_{2}, l_{2}, l_{3}, l_{6}, l_{6}, l_{6}\right)= \begin{cases}1 & \text { if } k \in[0,2) \\ 0.5 & \text { otherwise }\end{cases}
$$




\begin{tabular}{|c|c|c|c|c|}
\hline$k$ & $\frac{1}{m} \sum_{p=1}^{m} a_{12}^{p}$ & $\phi\left(\frac{1}{m} \sum_{p=1}^{m} a_{12}^{p}\right)$ & $\phi\left(\frac{s \cdot m+k}{2 m}\right)$ & $2 T M_{k}$ \\
\hline 0 & $(0+1+2+2+2+3+6+6+6) / 9=28 / 9$ & $\left(l_{3}, 1 / 9\right)$ & $\left(l_{3}, 0\right)$ & 1 \\
18 & $\left(l_{4}, 0\right)$ & 0.5 \\
\hline
\end{tabular}

Table 4: Aggregation and results for two different $2 T M_{k}-$ majorities

Examples 3 and 4 let us hypothesise that $L M_{K}$-majority and $2 T M_{k}$-majority coincide when the following relationship holds $K=k / s$. This will be proved in the following section.

\section{Equivalence between $L M_{K}$ and $2 T M_{k}$ majorities with difference in support}

So far, we have provided two apparently different extensions of $M_{k}$-majorities to the framework of the linguistic preferences. In this section, we prove that $L M_{K}$ and $2 T M_{k}$ are equivalent.

Let $l_{h} \in \mathcal{L}$ be a linguistic label, $\boldsymbol{u}\left(l_{h}\right)$ its associated centroid following the fuzzy set linguistic representation introduced in Subsection 2.3.1, and $a_{h}=\phi^{-1}\left(\left(l_{h}, 0\right)\right)$ the symbolic representation of the 2 -tuple $\left(l_{h}, 0\right)$ as defined in $(4)$. Let $\delta$ be the function that maps $a_{h}$ into $\boldsymbol{v}\left(l_{h}\right)$, i.e.

$$
\delta\left(a_{h}\right)=\boldsymbol{v}\left(l_{h}\right) .
$$

Under these conditions, $\delta$ as defined in (12) is the restriction of a continuous and strictly increasing function with domain $[0, s]$ :

$$
\delta:[0, s] \longrightarrow[0,1]
$$

such that $\delta(0)=0, \delta(s / 2)=0.5$ and $\delta(s)=1$.

Theorem 1 ( $L M_{K}$ and $2 T M_{k}$ Equivalence). If $\delta$ is additive then $L M_{K}$-majorities and $2 T M_{k}$-majorities are equivalent.

Proof. The following results is well known: if a continuous function verifies $F(x+y)=$ $F(x)+F(y) \forall x, y \in \mathbb{R}$ then there exists a constant $a \in \mathbb{R}$ such that $F(x)=a \cdot x \forall x \in \mathbb{R}$ [1]. This result applied to function $\delta$ implies that $\delta(x)=x / s$. Therefore we have:

$$
\frac{1}{m} \sum_{p=1}^{m} \boldsymbol{v}\left(\widetilde{A}_{i j}^{p}\right)>\frac{m+K}{2 m} \Leftrightarrow \frac{1}{m} \sum_{p=1}^{m} \delta\left(a_{i j}^{p}\right)>\frac{m+K}{2 m},
$$

i.e.

$$
\frac{1}{m} \sum_{p=1}^{m} \boldsymbol{v}\left(\widetilde{A}_{i j}^{p}\right)>\frac{m+K}{2 m} \Leftrightarrow \frac{1}{m} \sum_{p=1}^{m} a_{i j}^{p}>\frac{s \cdot m+s \cdot K}{2 m} .
$$

We conclude that $L M_{K}$-majorities is equivalent to $2 T M_{k}-$ majorities when $k=s \cdot K$.

Theorem 1 establishes the condition for $L M_{K}$-majorities and $2 T M_{k}$-majorities to be mathematically isomorphic: $\delta(x)=x / s$. Notice that in Subsection 3.1 we proved that $\boldsymbol{v}$ is additive and that under the assumption of symmetric distribution of the linguistic labels with respect to the central assessment it is $\boldsymbol{v}\left(l_{h}\right)=h / s$, which allows us to conclude that $L M_{K}$-majorities and $2 T M_{k}$-majorities are indeed equivalent when the membership 
functions of the fuzzy linguistic labels result in an evenly distribution of the centroids of the fuzzy linguistic labels. In the following section we prove a number of normative properties for the $2 T M_{k}$-majorities with difference in support, which obviously apply to the $L M_{K}$-majorities using the proved equivalence.

\section{Properties of linguistic majorities with difference in support}

For convenience, we use expression (10) for $2 T M_{k}$-majorities with difference in support:

$$
2 T M_{k}\left(a_{i j}^{1}, \ldots, a_{i j}^{p}\right)= \begin{cases}1 & \text { if } \frac{1}{m} \sum_{p=1}^{m} \phi\left(a_{i j}^{p}\right)>\phi\left(\frac{s \cdot m+k}{2 m}\right) \\ 0 & \text { if } \frac{1}{m} \sum_{p=1}^{m} \phi\left(a_{i j}^{p}\right)<\phi\left(\frac{s \cdot m-k}{2 m}\right) \\ 0.5 & \text { otherwise }\end{cases}
$$

where $\frac{1}{m} \sum_{p=1}^{m} \phi\left(a_{i j}^{p}\right)$ is the average of the 2-tuple representation of the linguistic preferences provided by the voters for the pair of alternatives $\left(x_{i}, x_{j}\right), \phi$ is the 2 -tuple symbolic aggregation mapping (3); and $k \in[0, m \cdot s)$ represents the threshold of support required for an alternative to be the social winner.

The first normative property says that $2 T M_{k}$-majorities fulfil anonymity, i.e. the order in which the linguistic valuations of the voters are given is irrelevant for the final social outcome. The proof is omitted because it is a direct consequence of the arithmetic mean being commutative.

Proposition 2 (Anonimity). Given a profile of linguistic preferences $\left(l_{i j}^{1}, \ldots, l_{i j}^{m}\right) \in \mathcal{L}^{m}$, the following equality holds

$$
2 T M_{k}\left(l_{i j}^{1}, \ldots, l_{i j}^{m}\right)=2 T M_{k}\left(l_{i j}^{\sigma(1)}, \ldots, l_{i j}^{\sigma(m)}\right) .
$$

for any permutation $\sigma:\{1, \ldots, m\} \rightarrow\{1, \ldots, m\}$.

Example 5. Given the profile of linguistic preferences $\left(l_{6}, l_{2}, l_{6}, l_{2}, l_{3}, l_{1}, l_{0}, l_{6}, l_{2}\right)$. Then we have

$$
\frac{1}{m} \sum_{p=1}^{m} a_{i j}^{p}=(6+2+6+2+3+1+0+6+2) / 9=28 / 9
$$

and

$$
\phi\left(\frac{1}{m} \sum_{p=1}^{m} a_{i j}^{p}\right)=\left(l_{3}, 1 / 9\right) .
$$

These values coincide with the result obtained in Example 4 and therefore it is

$$
2 T M_{k}\left(l_{6}, l_{2}, l_{6}, l_{2}, l_{3}, l_{1}, l_{0}, l_{6}, l_{2}\right)=2 T M_{k}\left(l_{0}, l_{1}, l_{2}, l_{2}, l_{2}, l_{3}, l_{6}, l_{6}, l_{6}\right) .
$$

Neutrality means that the aggregation rule should treat alternatives equally, which is proved in the following proposition.

Proposition 3 (Neutrality). Given a profile of linguistic preferences $\left(l_{i j}^{1}, \ldots, l_{i j}^{m}\right) \in \mathcal{L}^{m}$, the following equality holds

$$
2 T M_{k}\left(N\left(l_{i j}^{1}\right), \ldots, N\left(l_{i j}^{m}\right)\right)=1-2 T M_{k}\left(l_{i j}^{1}, \ldots, l_{i j}^{m}\right) .
$$


Proof. We have to prove the following three statements:

1. If $2 T M_{k}\left(N\left(a_{i j}^{1}\right), \ldots, N\left(a_{i j}^{m}\right)\right)=1$, then $2 T M_{k}\left(a_{i j}^{1}, \ldots, a_{i j}^{m}\right)=0$.

2. If $2 T M_{k}\left(N\left(a_{i j}^{1}\right), \ldots, N\left(a_{i j}^{m}\right)\right)=0$, then $2 T M_{k}\left(a_{i j}^{1}, \ldots, a_{i j}^{m}\right)=1$.

3. If $2 T M_{k}\left(N\left(a_{i j}^{1}\right), \ldots, N\left(a_{i j}^{m}\right)\right)=0.5$, then $2 T M_{k}\left(a_{i j}^{1}, \ldots, a_{i j}^{m}\right)=0.5$.

Given a profile of linguistic preferences, $\left(l_{i j}^{1}, \ldots, l_{i j}^{m}\right)$, expressed in terms of its equivalent symbolic translation, i.e. $\left(a_{i j}^{1}, \ldots, a_{i j}^{m}\right)$, we have

$$
2 T M_{k}\left(N\left(a_{i j}^{1}\right), \ldots, N\left(a_{i j}^{m}\right)\right)=1 \Leftrightarrow \frac{1}{m} \sum_{p=1}^{m} \phi\left(N\left(a_{i j}^{p}\right)\right)>\phi\left(\frac{s \cdot m+k}{2 m}\right) .
$$

Because $N\left(a_{i j}^{p}\right)=s-a_{i j}^{p}(\forall p)$ then

$$
\frac{1}{m} \sum_{p=1}^{m} \phi\left(N\left(a_{i j}^{p}\right)\right)>\phi\left(\frac{s \cdot m+k}{2 m}\right) \Leftrightarrow \frac{1}{m} \sum_{p=1}^{m} \phi\left(s-a_{i j}^{p}\right)>\phi\left(\frac{s \cdot m+k}{2 m}\right) .
$$

Recall that function $\phi$ is additive, and therefore it is

$$
\frac{1}{m} \sum_{p=1}^{m} \phi\left(s-a_{i j}^{p}\right)>\phi\left(\frac{s \cdot m+k}{2 m}\right) \Leftrightarrow \phi\left(\frac{1}{m} \sum_{p=1}^{m}\left(s-a_{i j}^{p}\right)\right)>\phi\left(\frac{s \cdot m+k}{2 m}\right) .
$$

Therefore we have:

$$
2 T M_{k}\left(N\left(a_{i j}^{1}\right), \ldots, N\left(a_{i j}^{m}\right)\right)=1 \Leftrightarrow \frac{1}{m} \sum_{p=1}^{m}\left(s-a_{i j}^{p}\right)>\frac{s \cdot m+k}{2 m} .
$$

Algebraic manipulation of the right hand side of this last equivalence yields:

$$
\frac{1}{m} \sum_{p=1}^{m}\left(s-a_{i j}^{p}\right)>\frac{s \cdot m+k}{2 m} \Leftrightarrow \frac{1}{m} \sum_{p=1}^{m} a_{i j}^{p}<\frac{s}{2}-\frac{k}{2 m} .
$$

Because $\phi$ is strictly increasing we have

$$
\frac{1}{m} \sum_{p=1}^{m} a_{i j}^{p}<\frac{s}{2}-\frac{k}{2 m} \Leftrightarrow \phi\left(\frac{1}{m} \sum_{p=1}^{m} a_{i j}^{p}\right)<\phi\left(\frac{s}{2}-\frac{k}{2 m}\right) .
$$

Finally, applying again the additivity property of function $\phi$ we conclude that $2 T M_{k}\left(N\left(a_{i j}^{1}\right), \ldots, N\left(a_{i j}^{m}\right)\right)=1 \Leftrightarrow \frac{1}{m} \sum_{p=1}^{m} \phi\left(a_{i j}^{p}\right)<\phi\left(\frac{s}{2}-\frac{k}{2 m}\right) \Leftrightarrow 2 T M_{k}\left(a_{i j}^{1}, \ldots, a_{i j}^{m}\right)=0$.

This proves item 1. The proofs of items 2 and 3 are similar.

Example 6. Given the profile of linguistic preferences $\left(l_{0}, l_{1}, l_{2}, l_{2}, l_{2}, l_{3}, l_{6}, l_{6}, l_{6}\right)$, then we have

$$
\left(N\left(l_{0}\right), N\left(l_{1}\right), N\left(l_{2}\right), N\left(l_{2}\right), N\left(l_{2}\right), N\left(l_{3}\right), N\left(l_{6}\right), N\left(l_{6}\right), N\left(l_{6}\right)\right)=\left(l_{6}, l_{5}, l_{4}, l_{4}, l_{4}, l_{3}, l_{0}, l_{0}, l_{0}\right) .
$$


Moreover,

$$
\frac{1}{m} \sum_{p=1}^{m} a_{i j}^{p}=(6+5+4+4+4+3+0+0+0) / 9=26 / 9
$$

and

$$
\phi\left(\frac{1}{m} \sum_{p=1}^{m} a_{i j}^{p}\right)=\left(l_{3},-1 / 9\right)
$$

Notice that $\phi\left(\frac{1}{m} \sum_{p=1}^{m} a_{i j}^{p}\right)>\phi\left(\frac{s \cdot m+k}{2 m}\right)$ never happens, no matter the value of $k$. On the other hand, when $k<2$ it is $\phi\left(\frac{1}{m} \sum_{p=1}^{m} a_{i j}^{p}\right)<\phi\left(\frac{s \cdot m-k}{2 m}\right)$. Consequently,

$$
2 T M_{k}\left(l_{6}, l_{5}, l_{4}, l_{4}, l_{4}, l_{3}, l_{0}, l_{0}, l_{0}\right)= \begin{cases}0 & \text { if } k \in[0,2) \\ 0.5 & \text { otherwise }\end{cases}
$$

Recalling the result of Example 4, the following holds:

$$
2 T M_{k}\left(l_{6}, l_{5}, l_{4}, l_{4}, l_{4}, l_{3}, l_{0}, l_{0}, l_{0}\right)=1-2 T M_{k}\left(l_{0}, l_{1}, l_{2}, l_{2}, l_{2}, l_{3}, l_{6}, l_{6}, l_{6}\right) .
$$

Monotonicity is proved next. Under this property, the majority value does not decrease when the individual linguistic preference evaluation of a profile increase.

Proposition 4 (Monotonicity). Given two profiles of linguistic preferences, $\left(l_{i j}^{1}, \ldots, l_{i j}^{m}\right)$ and $\left(l_{i j}^{\prime 1}, \ldots, l_{i j}^{\prime m}\right)$, such that it holds that $l_{i j}^{p} \geq l_{i j}^{p}(\forall p)$ then:

$$
2 T M_{k}\left(l_{i j}^{1}, \ldots, l_{i j}^{m}\right) \geq 2 T M_{k}\left(l_{i j}^{\prime}, \ldots, l_{i j}^{\prime m}\right) .
$$

Proof. Recall that both function $\phi$ and the arithmetic mean are increasing, and therefore denoting $l_{i j}^{p} \equiv \phi\left(a_{i j}^{p}\right)$ and $l_{i j}^{\prime p} \equiv \phi\left(a_{i j}^{\prime p}\right)$ we have

$$
l_{i j}^{p} \geq l_{i j}^{\prime p}(\forall p) \Rightarrow \frac{1}{m} \sum_{p=1}^{m} \phi\left(a_{i j}^{p}\right) \geq \frac{1}{m} \sum_{p=1}^{m} \phi\left(a_{i j}^{\prime p}\right),
$$

which proves that

$$
2 T M_{k}\left(l_{i j}^{1}, \ldots, l_{i j}^{m}\right) \geq 2 T M_{k}\left(l_{i j}^{\prime}, \ldots, l_{i j}^{\prime m}\right) .
$$

Example 7. Given the profile of linguistic preferences $\left(l_{3}, l_{1}, l_{2}, l_{2}, l_{2}, l_{3}, l_{6}, l_{6}, l_{6}\right)$. We have

$$
\frac{1}{m} \sum_{p=1}^{m} a_{i j}^{p}=31 / 9
$$

and

$$
\phi\left(\frac{1}{m} \sum_{p=1}^{m} a_{i j}^{p}\right)=\left(l_{3}, 4 / 9\right)
$$

Because $\phi\left(\frac{1}{m} \sum_{p=1}^{m} a_{i j}^{p}\right)>\phi\left(\frac{s \cdot m+k}{2 m}\right)$ when $k<8$, it is

$$
2 T M_{k}\left(l_{3}, l_{1}, l_{2}, l_{2}, l_{2}, l_{3}, l_{6}, l_{6}, l_{6}\right)= \begin{cases}1 & \text { if } k \in[0,8) \\ 0.5 & \text { otherwise }\end{cases}
$$

Consequently,

$$
2 T M_{k}\left(l_{3}, l_{1}, l_{2}, l_{2}, l_{2}, l_{3}, l_{6}, l_{6}, l_{6}\right) \geq 2 T M_{k}\left(l_{0}, l_{1}, l_{2}, l_{2}, l_{2}, l_{3}, l_{6}, l_{6}, l_{6}\right) .
$$


The weak Pareto property presented below, asserts that the result under the rule has to respect unanimous profiles.

Proposition 5 (Weak Pareto). The following equalities hold:

1. $2 T M_{k}\left(l_{s}, \ldots, l_{s}\right)=1$.

2. $2 T M_{k}\left(l_{0}, \ldots, l_{0}\right)=0$.

Proof.

1. We have

$$
\frac{1}{m} \sum_{p=1}^{m} \phi(s)=\phi(s)>\phi\left(\frac{m \cdot s+k}{2 m}\right) \forall k \in[0, m \cdot s),
$$

and therefore

$$
2 T M_{k}\left(l_{s}, \ldots, l_{s}\right)=1 \text {. }
$$

2. We observe that $\left(l_{0}, \ldots, l_{0}\right)=\left(N\left(l_{s}\right), \ldots, N\left(l_{s}\right)\right)$. Thus, the proof of this case is obvious from case 1 above and Proposition 3.

Example 8. Given the profile of linguistic preferences $\left(l_{6}, l_{6}, l_{6}, l_{6}, l_{6}, l_{6}, l_{6}, l_{6}, l_{6}\right)$ then we have $\frac{1}{m} \sum_{p=1}^{m} a_{i j}^{p}=6$ and $\phi\left(\frac{1}{m} \sum_{p=1}^{m} a_{i j}^{p}\right)=\left(l_{6}, 0\right)$. This is the highest collective preference valuation possible, and therefore it will be

$$
2 T M_{k}\left(l_{6}, l_{6}, l_{6}, l_{6}, l_{6}, l_{6}, l_{6}, l_{6}, l_{6}\right)=1 \forall k .
$$

Finally, the cancellative property is proved. Given two profiles with the same linguistic labels but two of them, then if the additions of the symbolic translations of the differing linguistic labels in each profile coincide, then the social majority is the same for the two profiles.

Proposition 6 (Cancellative). Given two profiles of linguistic preferences, $\left(l_{i j}^{1}, \ldots, l_{i j}^{m}\right)$ and $\left(l_{i j}^{\prime}, \ldots, l_{i j}^{\prime m}\right)$, such that

$$
l_{i j}^{h}=l_{i j}^{\prime h} \quad \forall h \neq o, q ; \quad l_{i j}^{o} \neq l_{i j}^{o}, l_{i j}^{q} \neq l_{i j}^{\prime q} \text { with } l_{i j}^{o}+l_{i j}^{q}=l_{i j}^{o}+l_{i j}^{\prime q}
$$

then

$$
2 T M_{k}\left(l_{i j}^{\prime}, \ldots, l_{i j}^{\prime m}\right)=2 T M_{k}\left(l_{i j}^{1}, \ldots, l_{i j}^{m}\right) .
$$

Proof. Notice that $l_{i j}^{h}=l_{i j}^{\prime h} \forall h \neq o, q ; \quad l_{i j}^{o} \neq l_{i j}^{\prime o}, l_{i j}^{q} \neq l_{i j}^{q}$ with $l_{i j}^{o}+l_{i j}^{q}=l_{i j}^{o}+l_{i j}^{q}$ implies $\frac{1}{m} \sum_{p=1}^{m} \phi\left(a_{i j}^{p}\right)=\frac{1}{m} \sum_{p=1}^{m} \phi\left(a_{i j}^{\prime p}\right)$.

Example 9. Given the following two profiles of linguistic preferences $\left(l_{3}, l_{1}, l_{2}, l_{2}, l_{2}, l_{3}, l_{6}, l_{6}, l_{3}\right)$ and $\left(l_{0}, l_{1}, l_{2}, l_{2}, l_{2}, l_{3}, l_{6}, l_{6}, l_{6}\right)$, then we have $\frac{1}{m} \sum_{p=1}^{m} a_{i j}^{p}=28 / 9$ and $\phi\left(\frac{1}{m} \sum_{p=1}^{m} a_{i j}^{p}\right)=\left(l_{3}, 1 / 9\right)$, for both of them. Therefore,

$$
2 T M_{k}\left(l_{3}, l_{1}, l_{2}, l_{2}, l_{2}, l_{3}, l_{6}, l_{6}, l_{3}\right)=2 T M_{k}\left(l_{0}, l_{1}, l_{2}, l_{2}, l_{2}, l_{3}, l_{6}, l_{6}, l_{6}\right) .
$$




\section{Conclusion}

'DEMOCRATIC THEORY is based on the premise that the resolution of a matter of social policy, group choice or collective action should be based on the desires or preferences of the individuals in the society, group or collective.' This quotation from Fishburn [10, page 3] fully justifies the use of preferences in social choice. However, democracy is recognised when decisions are made applying majority voting rule, which in its simple formulation means that the side with the most votes wins, whether it is an election, the select of the best candidate for a job when judged by a panel of experts, etc. This, however, is not the only majority rule available for social policy, group choice or collective action. Depending on the gravity or importance of the decision to make, other rules such as unanimous, absolute and qualified majorities may be more appropriate. These rules are easy to understand when each vote counts the same. However, there are many practical situations when this is not the case specially when experts or voters are allowed to indicate their degree of preference, which might be the case for example when selecting candidates for a job at a company. In these cases, it is possible to apply a new type of majority rules that are known as majorities based on difference in support, which allow to calibrate the amount of support required for the winner alternative by means of a difference of intensity of preference fixed before the election process. Majorities based on difference in support are defined when preferences are expressed quantitatively. This paper, however, deals with the comparison of two alternatives at a time and the experts or voters are allowed to express their preferences using qualitative ratings rather than quantitative ratings, a practice that is widely extended, for example, in market research for the introduction of new products by companies. This is because individuals, no mater their background, feel more comfortable using words to articulate their preferences than using numbers. In this context, the aim of this paper was to fill the gap between majorities based on difference of votes and majorities based on difference in support by providing new majority rules based on difference of support in the linguistic framework.

Linguistic majorities with difference in support extend majorities based on difference of votes from the context of crisp preferences to the framework of linguistic preferences. These linguistic majorities have been formalised for the two main representation methodologies of linguistic preferences: the cardinal, based on the use of fuzzy sets; and the ordinal, based on the use of the 2 -tuples. It has been proved that both representations are mathematically isomorphic when fuzzy numbers are ranked using their respective centroids, and therefore it can be concluded that the cardinal approach constitutes a more general framework to model linguistic majorities with difference in support. Finally, a set of normative properties have been demonstrated to hold for the new linguistic majorities.

Some interesting extensions are left opened. Among them, the study of the collective consistency of linguistic majorities with difference in support when more than two alternatives are compared [28], and the development of a consistency based selection process seems to be worth further investigation. Also, it seems interesting to explore softer approaches to linguistic majorities with difference in support when the information is represented using fuzzy sets. Both research issues mentioned here could be addressed by developing an alternative methodology to the use of a representative crisp number more consistent with the fuzzy nature of the linguistic information. A potential avenue to investigate could be the construction of a collective fuzzy preference structure [12] on the set of alternatives to represent the fuzzy linguistic majority with difference in support. Finally, the use of type-2 fuzzy sets also seems to be a challenging issue that deserves future research effort $[17,18]$, which can be an appropriate representation model of prefer- 
ences when different experts provide their information using sets of linguistic labels with different cardinality, a case that happens when experts have different levels of expertise. An alternative approach for this last scenario, which deserves further research, would be the application of aggregation operators able to aggregate fuzzy number not necessarily from the same set of linguistic labels, such as the type-1 OWA operator [6, 46].

\section{Acknowledgements}

Part of this research is the result of Patrizia Pérez-Asurmendi's research visit to the DMU Interdisciplinary Group in Intelligent Transport Systems at De Montfort University (Leicester, UK) in the autumn of 2012. The authors are grateful to José Luis García-Lapresta and Bonifacio Llamazares, as well as the four anonymous reviewers and the managing editor of Applied Soft Computing, for their valuable comments and suggestions that have helped us to improve considerably the quality of this paper. This work is partially supported by the Spanish Ministry of Science and Innovation (Projects ECO2009-07332, ECO2012-32178 and ECO2012-34202) and ERDF.

[1] T. M. Apostol. Mathematical analysis. 2nd Edition. Addison-Wesley, Massachusetts, 1974.

[2] J. Bezdek, B. Spillman, R. Spillman. "A fuzzy relation space for group decision theory", Fuzzy Sets and Systems, 1, 255-268, 1978.

[3] M. Brunelli, J. Mezei. "How different are ranking methods for fuzzy numbers? A numerical study", International Journal of Approximate Reasoning, 54 (5), 627-639, 2013.

[4] S.Chen, C. Hwan. Fuzzy multiple attribute decision making-methods and applications. Springer, Berlin, 1992.

[5] F. Chiclana, E. Herrera-Viedma, S. Alonso, F. Herrera. "Cardinal consistency of reciprocal preference relations: a characterization of multiplicative transitivity", IEEE Transactions on Fuzzy Systems, 17 (1), 14-23, 2009.

[6] F. Chiclana, S. M. Zhou. "Type-reduction of general type-2 fuzzy sets: The type-1 OWA approach", International Journal of Intelligent Systems, 28 (5), 505-522, 2013.

[7] M. Delgado, J.L. Verdegay, M.A. Vila. "Linguistic decision making models", International Journal of Intelligent Systems, 7, 479-492, 1992.

[8] M. Delgado, J.L. Verdegay, M.A. Vila. "On aggregation operations of linguistic labels", International Journal of Intelligent Systems, 8 (3), 351-370, 1993.

[9] J.A. Ferejohn, D.M. Grether. "On a class of rational social decisions procedures", Journal of Economic Theory, 8, 471-482, 1974.

[10] P.C. Fishburn. The Theory of Social Choice. Princeton University Press, Princeton, 1973.

[11] P.C. Fishburn. "Preference structures and their numerical representations", Theoretical Computer Science, 217, 359-383, 1999. 
[12] J. Fodor, M. Roubens. Fuzzy preference modelling and multicriteria decision support. Kluwer Academics Publishers, Dordrecht, 1994.

[13] J.L. García-Lapresta. "A general class of simple majority decision rules based on linguistic opinions", Information Sciences, 176 (4), 352-365, 2006.

[14] J.L. García-Lapresta, B. Llamazares. "Majority decisions based on difference of votes", Journal of Mathematical Economics, 35, 463-481, 2001.

[15] J.L. García-Lapresta, B. Llamazares. "Preference intensities and majority decisions based on difference of support between alternatives", Group Decision and Negotiation, 19, 527-542, 2010.

[16] J.L. García-Lapresta, B. Llamazares, M. Martínez-Panero. "A social choice analysis of the Borda rule in a general linguistic framework", International Journal of Computational Intelligence Systems, 3, 501-513, 2010.

[17] S. Greenfield, F. Chiclana. "Accuracy and complexity evaluation of defuzzification strategies for the discretised interval type-2 fuzzy set", International Journal of Approximate Reasoning, 54 (8), 1013-1033, 2013.

[18] S. Greenfield, F. Chiclana. "Defuzzification of the Discretised Generalised Type-2 Fuzzy Set: Experimental Evaluation", Information Sciences, 224, 1-25, 2013.

[19] M. Hanss. Applied Fuzzy Arithmetic. An Introduction with Engineering Applications. Springer-Verlag, Berlin-Heidelberg, 2005.

[20] F. Herrera, E. Herrera-Viedma, J.L. Verdegay. "A linguistic decision process in group decision making", Group Decision and Negotiation, 5, 165-176, 1996.

[21] F. Herrera, L. Martínez. "A 2-tuple fuzzy linguistic representation model for computing with words", IEEE Transactions on Fuzzy Systems, 8, 746-752, 2000.

[22] F. Herrera, S. Alonso, F. Chiclana, E.Herrera-Viedma. "Computing with words in decision making: foundations, trends and prospects", Fuzzy Optimization and Decision Making, 8, 337-364, 2009.

[23] N. Houy. "Some further characterizations for the forgotten voting rules", Mathematical Social Sciences, 53, 111-121, 2007.

[24] J. Kacprzyk. "Group decision making with a fuzzy linguistic majority", Fuzzy Sets and Systems, 18, 105-118, 1986.

[25] J. Kacprzyk, M. Fedrizzi. Multiperson decision making models using fuzzy sets and possibility theory. Kluwer Academic Publishers, Dordrecht, 1990.

[26] J. Kacprzyk, M. Fedrizzi, H. Nurmi. "Group decision making and consensus under fuzzy preferences and fuzzy majority", Fuzzy Sets and Systems, 49, 21-31, 1992.

[27] B. Llamazares. "The forgotten decision rules: Majority rules based on difference of votes", Mathematical Social Sciences, 51, 311-326, 2006.

[28] B. Llamazares, P. Pérez-Asurmendi, J.L. García-Lapresta. "Collective transitivity in majorities based on difference in support", Fuzzy Sets and Systems, 216, 3-15, 2013. 
[29] J. Lu, G. Zhang, D. Ruan. F. Wu. Multi-objective group decision making. Methods, software and applications with fuzzy set techniques. Imperial College Press, London, 2007.

[30] R.D. Luce and P. Suppes. "Preferences, Utility and Subject Probability", in: R.D. Luce et al. (Eds.) Handbook of Mathematical Psychology, Vol. III, Wiley, New York, 249-410, 1965.

[31] K.O. May. "A set of independent necessary and sufficient conditions for simple majority decisions", Econometrica, 20, 680-684, 1952.

[32] I. Millet. "The effectiveness of alternative preference elicitation methods in the analytic hierarchy process", Journal of Multi-Criteria Decision Analysis, 6, 41-51, 1997.

[33] H. Nurmi. "Approaches to collective decision making with fuzzy preference relations", Fuzzy Sets and Systems, 6, 249-259, 1981.

[34] H. Nurmi. "Fuzzy social choice: A selective retrospect", Soft Computing, 12, 281$288,2008$.

[35] M. Roubens, P. Vincke. Preference modelling. Lecture Notes in Economics and Mathematical Systems, 250. Springer, Berlin, 1985.

[36] D.G. Saari. "Consistency of decision processes", Annals of Operations Research, 23, 103-137, 1990.

[37] T.L. Saaty. The Analytic Hierarchy Process. McGraw-Hill, New York, 1980.

[38] A.K. Sen. Collective Choice and Social Welfare. Holden-Day, San Francisco, 1970.

[39] Z. Xu. "A method based on linguistic aggregation operators for group decision making with linguistic preference relations", Information Sciences, 166 (1-4), 19-30, 2004 .

[40] R. Yager. "A new methodology for ordinal multiobjective decisions based on fuzzy sets", Decision Sciences, 12, 589-600, 1981.

[41] R. Yager. "OWA aggregation over a continuous interval argument with applications to decision making", IEEE Transactions on Systems, Man and Cybernetics, Part B: Cybernetics, 34 (4), 1952-1963, 2004.

[42] L.A. Zadeh. "Fuzzy sets", Information and Control, 8 (3), 338-357, 1965.

[43] L.A. Zadeh. "Outline of a new approach to the analysis of complex systems and decision processes", IEEE Transactions on Systems, Man and Cybernetics, 3, 28-44, 1973.

[44] L.A. Zadeh. "The concept of a linguistic variable and its application to approximate reasoning-I", Information Sciences, 8, 199-249, 1975.

[45] L.A. Zadeh. "The concept of a linguistic variable and its application to approximate reasoning-II", Information Sciences, 8, 301-357, 1975. 
[46] S.M. Zhou, F. Chiclana, R.I. John, J.M. Garibaldi. "Type-1 OWA operators for aggregating uncertain information with uncertain weights induced by type-2 linguistic quantifiers", Fuzzy Sets and Systems, 159 (24), 3281-3296, 2008.

[47] S.M. Zhou, F. Chiclana, R.I. John, J.M. Garibaldi. "Alpha-level aggregation: A practical approach to Type-1 OWA operation for aggregating uncertain information with applications to breast cancer treatments", IEEE Transactions on Knowledge and Data Engineering, 23 (10), 1455-1468, 2011. 